\title{
Multi-instrument observations of the solar eclipse on 20 March 2015 and its effects on the ionosphere over Belgium and Europe
}

\author{
Stanimir M. Stankov ${ }^{1,4},{ }^{*}$, Nicolas Bergeot ${ }^{1,2}$, David Berghmans ${ }^{1,2}$, David Bolsée ${ }^{1,3}$, Carine Bruyninx ${ }^{1,2}$, \\ Jean-Marie Chevalier ${ }^{1,2}$, Frédéric Clette ${ }^{1,2}$, Hugo De Backer ${ }^{1,4}$, Johan De Keyser ${ }^{1,3}$, Elke D’Huys ${ }^{1,2}$, \\ Marie Dominique ${ }^{1,2}$, Joseph F. Lemaire ${ }^{1,3}$, Jasmina Magdalenic ${ }^{1,2}$, Christophe Marqué ${ }^{1,2}$, Nuno Pereira ${ }^{1,3}$, \\ Viviane Pierrard $^{1,3}$, Danislav Sapundjiev ${ }^{1,4}$, Daniel B. Seaton ${ }^{1,2}$, Koen Stegen ${ }^{1,2}$, Ronald Van der Linden ${ }^{1,2}$, \\ Tobias G.W. Verhulst ${ }^{1,4}$, and Matthew J. West ${ }^{1,2}$ \\ Solar-Terrestrial Centre of Excellence (STCE), Ringlaan 3, 1180 Brussels, Belgium \\ Royal Observatory of Belgium (ROB), Ringlaan 3, 1180 Brussels, Belgium \\ 3 Royal Belgian Institute for Space Aeronomy (BISA), Ringlaan 3, 1180 Brussels, Belgium \\ 4 Royal Meteorological Institute (RMI), Ringlaan 3, 1180 Brussels, Belgium \\ *Corresponding author: s.stankov@meteo.be
}

Received 25 July 2016 / Accepted 3 July 2017

\begin{abstract}
A total solar eclipse occurred on 20 March 2015, with a totality path passing mostly above the North Atlantic Ocean, which resulted in a partial solar eclipse over Belgium and large parts of Europe. In anticipation of this event, a dedicated observational campaign was set up at the Belgian Solar-Terrestrial Centre of Excellence (STCE). The objective was to perform high-quality observations of the eclipse and the associated effects on the geospace environment by utilising the advanced space- and ground-based instrumentation available to the STCE in order to further our understanding of these effects, particularly on the ionosphere. The study highlights the crucial importance of taking into account the eclipse geometry when analysing the ionospheric behaviour during eclipses and interpreting the eclipse effects. A detailed review of the eclipse geometry proves that considering the actual obscuration level and solar zenith angle at ionospheric heights is much more important for the analysis than at the commonly referenced Earth's surface or at the plasmaspheric heights. The eclipse occurred during the recovery phase of a strong geomagnetic storm which certainly had an impact on (some of) the ionospheric characteristics and perhaps caused the omission of some "low-profile" effects. However, the analysis of the ionosonde measurements, carried out at unprecedented high rates during the eclipse, suggests the occurrence of travelling ionospheric disturbances (TIDs). Also, the high temporal and spatial resolution measurements proved very important in revealing and estimating some finer details of the delay in the ionospheric reaction and the ionospheric disturbances.
\end{abstract}

Key words. Sun - Solar eclipse - Eclipse geometry - Ionosphere - Irregularities

\section{Highlights}

- High-resolution temporal and spatial measurements needed for detailed analyses.

- Eclipse geometry is of crucial importance for reliable interpretation of results.

- Ionosonde measurements indicate eclipse-generated wave phenomena.

\section{Introduction}

A solar eclipse occurs when the Moon passes between the Sun and the Earth, obscuring the Sun from view on part of the Earth's surface, totally or partially. Solar eclipses attract attention as these relatively rare conditions offer opportunities for advanced solar, ionospheric and atmospheric research.

Total solar eclipses provide unique conditions for observation of the Sun's outer atmospheric layers, the solar corona, chromosphere and prominences. Eclipse observations led to several fundamental discoveries, such as the first identification of a new chemical element (Helium), the first physical proof of general relativity (by measuring the deflection of light from background stars by the Sun's mass) and the extremely high temperature (exceeding 1,000,000 K) of the corona. Over the years, the Royal Observatory of Belgium (ROB) has carried out scientific expeditions to several locations around the world where (total) eclipses could be observed from the ground (Koeckelenbergh 1999). Primary objectives of the missions included the determination of coronal electron density, gravimetric and meteorological measurements (e.g. Clette et al. 1985, 1994; Gabryl et al. 1997, 1999).

Solar eclipse observations can be particularly useful when studying the ionosphere (Beynon \& Brown 1956). During eclipses, the quick changes in the solar electromagnetic flux and in the ionising ultraviolet (UV) radiation produce various effects on both the thermosphere and the ionosphere, including a modification of the temperature balance, production and loss of ionisation in the lower ionosphere, an alteration of the transport processes in the upper ionosphere, etc. (Rishbeth 1968, 1970). 
Earlier investigations of eclipse effects on the ionosphere were carried out by observing the changes in intensity of radio waves reflected from the ionosphere, followed by absorption measurements at oblique incidence on one or multiple frequencies, vertical incidence sounding (VIS) with ionosondes (Farges et al. 2001), in situ measurements with rockets and satellites, total electron content (TEC) deduced from the Faraday rotation in the polarisation of lunar radio waves (Klobuchar \& Whitney 1965), and more recently, with the advancement of the Global Navigation Satellite System (GNSS) technology, via GNSS-based TEC observations. Simultaneous multi-instrument observations (at single and/or several locations) (Farges et al. 2001; Jakowski et al. 2008) and targeted modelling studies (Müller-Wodarg et al. 1998) seem to be most efficient when studying the complex, multifaceted nature of solar eclipses and their effects on the ionosphere.

While, initially, efforts went into investigating the source of atmospheric ionisation (electromagnetic or corpuscular radiation) and chemical composition of the upper atmosphere (e.g. ionospheric plasma recombination rates), the interest shifted in recent years towards investigating the atmospheric gravity wave (AGW) phenomena generated by the Moon's shadow passing (at a supersonic speed) through the atmosphere (Chimonas \& Hines 1970; Altadill et al. 2001; Davis et al. 2001; Sauli et al. 2006, 2007; Jakowski et al. 2008).

This paper presents observations of the total solar eclipse that occurred on 20 March 2015. The first contact of the penumbra occurred in the Mid-Atlantic Ocean, north of the equator, at 07:40:52 UT and its last contact was in Central Asia at 11:50:13 UT. The greatest eclipse occurred at 09:45:39 UT at a location with coordinates of $64.43^{\circ} \mathrm{N}, 6.65^{\circ} \mathrm{W}$, where the path width was $462.6 \mathrm{~km}$. Most of the total eclipse path was above the North Atlantic Ocean, ending above the Arctic Ocean (Fig. 1A). As a result, a partial solar eclipse was visible from Belgium, including the STCE observation sites in Brussels, Dourbes and Humain (see Table 1 for the eclipse characteristics at these locations). At ground level, the maximum obscuration above Europe (Fig. 1B) ranged between about $95 \%$ in the North-West (NW) and about $20 \%$ in the South-East (SE) and the solar zenith angle (Fig. 1C) was between $70^{\circ}(\mathrm{NW})$ and $35^{\circ}(\mathrm{SE})$.

The paper presents eclipse observations carried out by the Belgian Solar-Terrestrial Centre of Excellence (STCE) with various instruments at its disposal. The purpose is to review the current knowledge and understanding of the eclipse effects and provide a detailed analysis of the eclipse effects on the ionosphere over Belgium and Europe, specific for this eclipse. Modelling results, involving reconstruction of the plasmasphere densities and temperatures as well as the ionospheric plasma redistribution, are also presented and discussed. The eclipse geometry and the influence it has on the ionospheric behaviour during the eclipse are also provided in great detail.

The paper is organised as follows. First, the instrumentation and measurement techniques used in this study are presented, followed by a description of the models and operational monitoring systems developed by the STCE and used for investigating the various phenomena associated with the eclipse. Next, the background conditions before and during the eclipse are described. The following section presents the results of the solar and plasmasphere-ionosphere observations and modelling during both the eclipse day and a control day
(A)

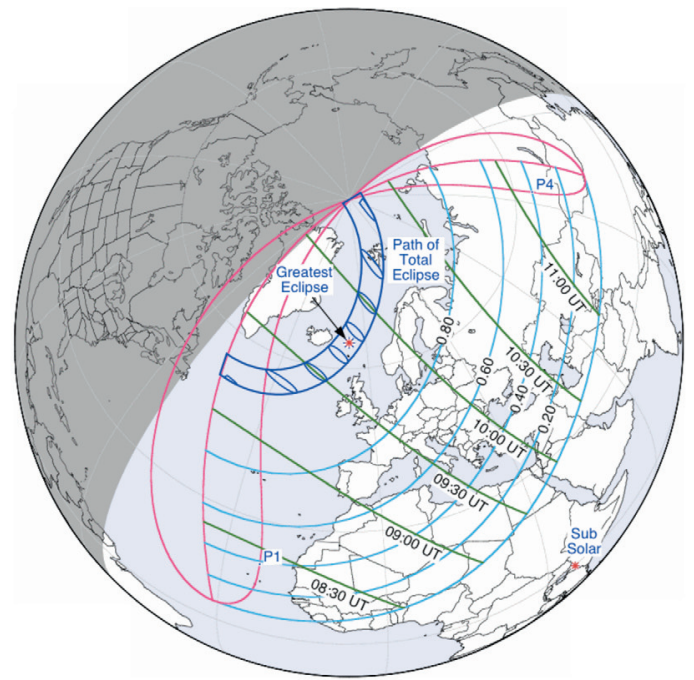

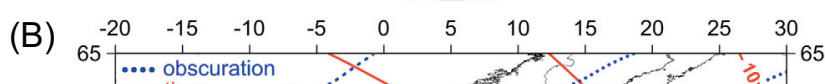
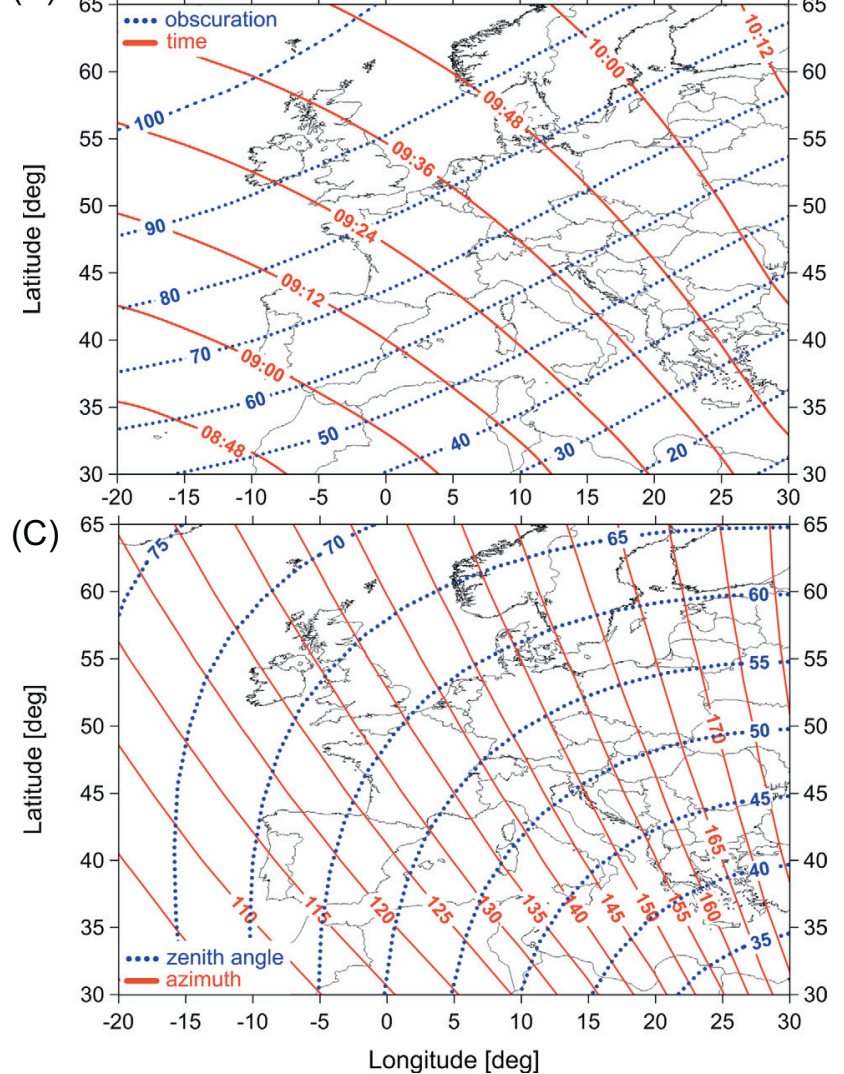

Fig. 1. Eclipse geometry at sea level. (A) Schematic (credit: NASA) of the solar eclipse on 20 March 2015 at the Earth's surface. The path of the total solar eclipse is denoted by the strip bounded by dark blue curves, while paths of the partial $(0.80,0.60,0.40$, and 0.20 magnitude) eclipse are plotted with light blue curves. The progression of the greatest eclipse is marked by green curves with time stamps (UT). (B) Times of the maximum eclipse (solid red lines) and maximum obscuration levels (dotted blue lines) over Europe. (C) Azimuth (solid red lines) and zenith angle (dotted blue lines) at the maximum eclipse over Europe. Note: Cylindrical equidistant mapping is applied in (B) and (C).

of normal conditions. In the next sections, the ionospheric observations are further analysed in search of wavelike structures, effects of the eclipse geometry are discussed and the 
Table 1. Eclipse characteristics (time, magnitude, maximal obscuration, elevation and azimuth) for the main observational sites in Belgium Brussels, Dourbes and Humain.

\begin{tabular}{|c|c|c|c|c|c|c|c|c|c|c|c|c|}
\hline \multirow[t]{2}{*}{ Location } & \multirow{2}{*}{$\begin{array}{c}\text { Coordinates } \\
{\left[{ }^{\circ} \mathrm{N},{ }^{\circ} \mathrm{E}\right]}\end{array}$} & \multicolumn{3}{|c|}{ Time $(\mathrm{UT})$} & \multirow{2}{*}{$\begin{array}{c}\text { Magnitude } \\
\text { Max } \\
\end{array}$} & \multirow{2}{*}{$\begin{array}{c}\text { Obscuration } \\
\text { Max (\%) }\end{array}$} & \multicolumn{3}{|c|}{ Elevation [degree] } & \multicolumn{3}{|c|}{ Azimuth [degree] } \\
\hline & & Start & $\operatorname{Max}$ & End & & & Start & Max & End & Start & Max & End \\
\hline Brussels & $50.8,4.4$ & $08: 27$ & $09: 35$ & $10: 45$ & 0.830 & 79.5 & 23.4 & 31.4 & 37.2 & 122.5 & 139.2 & 159.5 \\
\hline Dourbes & $50.1,4.6$ & $08: 26$ & $09: 34$ & $10: 45$ & 0.818 & 78.1 & 23.8 & 32.0 & 37.9 & 122.3 & 138.9 & 159.4 \\
\hline Humain & $50.2,5.3$ & $08: 27$ & $09: 35$ & $10: 46$ & 0.815 & 77.7 & 24.2 & 32.3 & 38.0 & 123.2 & 140.0 & 160.7 \\
\hline
\end{tabular}

results are compared with those from the previous eclipse in Europe in October 2005. The paper concludes with a summary of all results and an outlook for further investigations.

\section{Instrumentation and measurement techniques}

\subsection{Uccle Solar Equatorial Table (USET) telescope}

The Uccle Solar Equatorial Table (USET) (http://sidc.be/uset/ usetpres.php), located at the ROB in Brussels, carries four independent refracting telescopes (Berghmans et al. 2005):

- A $150 \mathrm{~mm}$ white-light telescope that projects a $25 \mathrm{~cm}$ image of the photosphere on a screen for visual sunspot drawings. Those drawings allow the long-term cataloguing of sunspot groups and the determination of the sunspot number, $S_{N}$.

- A $150 \mathrm{~mm}$ white-light telescope for photospheric CCD (charge-coupled devices) imaging (broad spectral bandpass in the blue-green, at $\lambda_{\max }=510 \mathrm{~nm}$, band: $415-573 \mathrm{~nm})$.

- An $80 \mathrm{~mm} \mathrm{H \alpha}$ telescope for CCD imaging of the chromosphere, equipped with a $0.05 \mathrm{~nm}$ Fabry-Pérot monochromator centred on the $\mathrm{H} \alpha$ Balmer line of Hydrogen $(656.280 \mathrm{~nm})$. This is the standard bandpass for the monitoring of solar flares in active regions and of the evolution and the eruptions of solar prominences.

- A $132 \mathrm{~mm}$ Calcium-line telescope for CCD imaging of the solar chromosphere, equipped with a $0.27 \mathrm{~nm}$ narrow-band interference filter centred on the CaII-K line $(393.37 \mathrm{~nm})$. This bandpass is adapted both for solar flare monitoring and for the mapping of bright chromospheric emission in the solar plages and the chromospheric network that determine the strong variations of solar irradiance in the ultraviolet.

\subsection{SWAP and LYRA instruments onboard PROBA2 satellite}

PROBA2 (Project for Onboard Autonomy) is an ESA (European Space Agency) micro-satellite launched in November 2009 hosting 17 new technological developments and 4 scientific instruments. PROBA2 began its scientific mission in March 2010. The spacecraft has a low Sunsynchronous Earth orbit (altitude of $725 \mathrm{~km}$ ), providing nearcontinual coverage of the Sun. The science payload onboard PROBA2 consists of two main solar instruments, Sun Watcher with Active Pixels and Image Processing (SWAP) and LYRA (Large Yield Radiometer, formerly Lyman- $\alpha$ Radiometer), and two instruments to observe the space environment in the immediate vicinity of the spacecraft, DSLP (Dual Segmented Langmuir Probe) and TPMU (Thermal Plasma Measurement
Unit). With these instruments, the aim is to identify and study all events on the Sun that might have implications on the solarterrestrial connection, both through imaging (SWAP) as well as through irradiance measurements (LYRA). In particular, the focus of the PROBA2 mission is the genesis and evolution of events that can affect space weather, such as coronal mass ejections (CMEs), waves and dimmings, solar flares, etc.

The Sun Watcher with Active Pixels and Image Processing (SWAP) solar telescope (Halain et al. 2013; Seaton et al. 2013) onboard the PROBA2 spacecraft observes the solar corona in a bandpass near $17.4 \mathrm{~nm}$ with a field of view $54 \times 54$ arcmin, roughly 1.7 solar radii along the image axes and 2.5 solar radii on the diagonals, providing the widest-field images of the Extreme Ultraviolet (EUV) corona available from the Earth's perspective.

LYRA (Large Yield Radiometer, formerly Lyman- $\alpha$ Radiometer) (Dominique et al. 2013) is an X-ray/ultraviolet radiometer that observes the full-Sun in four broad passbands, chosen for their relevance to solar physics, aeronomy and space weather: 1-20 nm, 1-80 nm, 120-123 nm, 190-222 nm. Those passbands are replicated in the three redundant units that constitute the instrument. Amongst those units, one is continuously in use, but is therefore affected by strong degradation, especially of the two longer wavelength channels, one is used for occasional observation campaigns and one is for calibration. The nominal acquisition cadence is $20 \mathrm{~Hz}$ and can go up to $100 \mathrm{~Hz}$. The primary scientific target of this instrument is the observation and analysis of flares, for which its wavelength ranges, high cadence and high signal-to-noise ratio are valuable assets.

\subsection{Solar spectrum on the SOLAR platform onboard ISS (SOLAR/SOLSPEC)}

SOLSPEC (Solar Spectrum on SOLAR platform), installed on the SOLAR platform of the COLUMBUS module onboard the International Space Station (ISS) since 2008, is an instrument designed to provide an accurate measurement of the extraterrestrial solar spectral irradiance (SSI) (Thuillier et al. 2009) in which the Royal Belgian Institute for Space Aeronomy (BISA) plays an important role. SOLSPEC is composed of three measurement channels covering a spectral range from $155 \mathrm{~nm}$ to $3088 \mathrm{~nm}$ - UV (229 nm), Vis $(473 \mathrm{~nm})$ and IR $(1575 \mathrm{~nm})$. It is absolutely calibrated against a primary standard of spectral irradiance and it possesses an internal lamp calibration unit, used to monitor the instrument's degradation during the mission.

\subsection{Solar radio telescope}

The ROB monitors the solar activity at radio wavelengths with a $6-\mathrm{m}$ dish radio telescope located at Humain $\left(50.2^{\circ} \mathrm{N}, 5.3^{\circ} \mathrm{E}\right)$. Observations are performed from sunrise to sunset irrespective 
of weather conditions. At the time of the eclipse on 20 March 2015, two CALLISTO (Compound Astronomical Low-cost Low-frequency Instrument for Spectroscopy and Transportable Observatory) spectrometers were in operation: one covering the frequency range $45-440 \mathrm{MHz}$ and another covering the 270-870 MHz range. They were connected to a broadband log-periodic antenna mounted as a piggy-bag on the side of the dish and to a dual-polarised log-periodic antenna placed at the focus point of the parabolic dish. Only the instrument covering the $270-870 \mathrm{MHz}$ range is used in this study, capable of observing the variation of the "quiet Sun" level during the eclipse and the recovery to normal conditions afterwards. The instrument is part of the e-CALLISTO network (http://e-callisto.org).

\subsection{GNSS receivers and network}

A NovAtel GPStation- $6^{(\mathrm{TM})}$ receiver is used for GNSS TEC measurements at the Royal Meteorological Institute (RMI) Geophysical Centre in Dourbes $\left(50.1^{\circ} \mathrm{N}, 4.6^{\circ} \mathrm{E}\right)$. The receiver can track all present and upcoming GNSS constellations and satellite signals with a maximum sampling rate of $50 \mathrm{~Hz}$ for each of the 120 available tracking channels. Also, continuous GNSS observations are carried out by the ROB at different locations in Belgium. In addition, GNSS observations from the EUREF Permanent GNSS Network (EPN) (Bruyninx et al. 2012) were also used in this study. Currently, the EUREF Network consists of more than 310 multi-GNSS stations, providing GNSS data in the standard RINEX (Receiver Independent Exchange) format with a 30-s sampling rate. The majority of the stations $(98 \%)$ provide daily data and $47 \%$ of them provide data in real time.

\subsection{Digital ionospheric sounder}

The lower ionosphere is predominantly monitored with highfrequency (HF) ionospheric sounders. The instrument used in this study is a Lowell Digisonde-4D ${ }^{\circledR}$ (Reinisch et al. 2009), installed at the RMI Geophysical Centre in Dourbes (Jodogne \& Stankov 2002). Digisonde-4D is a state-of-the-art equipment using HF radar remote-sensing principles to evaluate, with high accuracy and precision, the conditions of the ionospheric plasma above the station. It is capable of simultaneously measuring the following observables using reflected (in vertical incidence) or refracted (in oblique incidence) signals from the ionosphere: frequency, range, amplitude, phase, Doppler shift and spread, angle of arrival and wave polarisation. Signal transmission is performed with two (NE-SW and NW-SE) crossed "delta" antennas of $40 \mathrm{~m}$ in height, and reception is done with an array of four crossed magnetic dipole antennas in a triangular arrangement. The Digisonde is equipped with the latest version of the computer software for automatic ionogram interpretation, ARTIST-5 (Automatic Real-Time Ionogram Scaler with True height) (Galkin et al. 2008).

\section{3. lonosphere monitoring systems}

\subsection{Regional ionosphere monitoring and mapping (ROB-IONO)}

The ROB-IONO software (Bergeot et al. 2014) is used in this study to re-process GPS (Global Positioning System) and GLONASS (Global Navigation Satellite System, Russia) data from up to 280 stations available in 2015 from the EUREF network. The output consists of (European) regional maps of vertical TEC (in TEC units, 1 TECU $=10^{16} \mathrm{el} / \mathrm{m}^{2}$ ). Nominally, the maps are produced in near-real time every $15 \mathrm{~min}$ on $0.5^{\circ} \times 0.5^{\circ}$ grids extending from $-20^{\circ}$ to $+30^{\circ}$ in longitude and $32^{\circ}$ to $65^{\circ}$ in latitude. However, upon necessity, as for this study, higher-cadence (5-min) maps were produced.

\subsection{Local ionosphere monitoring and visualisation (LIEDR)}

An operational system has been developed and installed at the RMI with the purpose of measuring and displaying the local ionosphere characteristics, including the TEC, the ionospheric layers' critical frequencies and density peak heights, the plasma scale height, the slab thickness, the ionospheric tilt and plasma drift, etc. LIEDR (Local Ionospheric Electron Density Profile Reconstruction) is the main module used for retrieving and processing data in real time from simultaneous GNSS and ionosonde measurements and then constructing the vertical plasma density distribution (profile) in the local ionosphere (Stankov et al. 2011). The resulting full-height ionospheric electron density profile is displayed in the form of a "profilogram" at a (nominal) 5-min update rate. Another module is concerned with monitoring the ionospheric slab thickness (Stankov \& Warnant 2009), an important characteristic offering substantial information on the shape of the electron density profile, on the ionospheric composition and dynamics, etc. Ionospheric tilt and plasma drifts are distinctive features of the ionosphere related to its complex structures and irregularities that adversely affect radio-wave propagation. The tilt and drifts are monitored via ionosonde measurements and can also be used for studying the eclipse effects (Verhulst et al. 2016).

\section{Background conditions prior to and during the eclipse}

This section presents the "background" geospace conditions during the week of 13-19 March 2015 preceding the eclipse to ensure that the underlying processes able to influence the ionosphere are properly accounted for in the analysis of the eclipse observations. In particular, disturbances in the geomagnetic field, and in the magnetosphere in general, are well known for affecting the (diffusive and convective) transport processes in the topside ionosphere and plasmasphere. The analysis of these background conditions will help in selecting a "control" day providing a reference to the regular, unperturbed state of the ionosphere.

The solar eclipse on 20 March 2015 occurred during a period of rather low solar activity in February-March 2015 and within a short dip in solar activity (Fig. 2A), $S_{N}=21$ (20 March), between two substantial increases up to about $S_{N}=75$ (12 March) and $S_{N}=107$ (24 March). This variation matches the modulation of the CaII chromospheric emission over the two weeks framing the eclipse. The concentrations of activity at two opposite longitudes induced a 14-day (half-solar rotation) modulation in $S_{N}$ that persisted from mid-February to early April. During the week before the eclipse, between 13 and 19 March 2015, US National Oceanic and Atmospheric Administration (NOAA) Active Region (AR) 12297 was the major source of solar activity. This region traversed from the disk centre towards the West solar limb and produced seven M-class flares and a number of C-class flares. The largest one was an M1.8 class flare produced on 13 March 2015 peaking at 06:07 UT. Several of the flares produced by AR 12297 were 


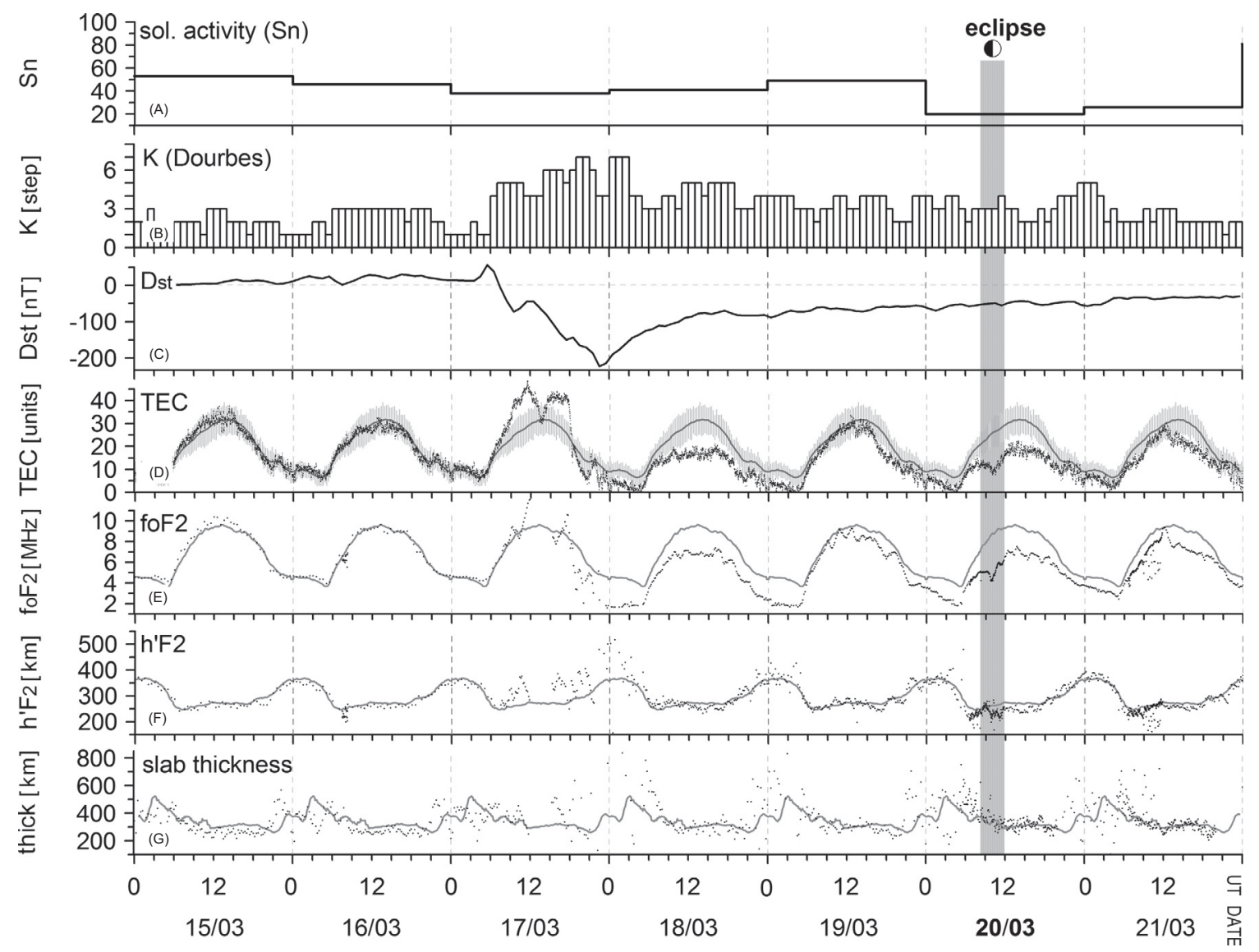

Fig. 2. Solar, geomagnetic and local ionospheric activity (Dourbes) measurements during the period 15-21 March 2015: Solar sunspot index, $S_{N}(\mathrm{~A})$, local magnetic index $K$ at Dourbes (B), Dst index (C), GPS TEC (D), $f_{o} \mathrm{~F}_{2}(\mathrm{E}), h^{\prime} \mathrm{F}_{2}(\mathrm{~F})$ and ionospheric slab thickness $(\mathrm{G})$. Instantaneous measurements denoted with dots and the 27-day medians (based on data from 4 to 30 March) plotted with solid (grey) curves. The standard deviation $(2 \sigma)$, centred on the median, is given by vertical (grey) bars.

associated with Coronal Mass Ejections (CMEs), dimmings and EUV waves. However, AR 12297 activity decreased towards the day of the eclipse and the region did not produce greater than C-class flares after 18 March 2015. The solar flux index F10.7 was between 110 and 120 units.

A massive interplanetary CME, associated with the C9.1 flare from NOAA AR 12297, was detected by the Solar and Heliospheric Observatory satellite/Large Angle and Spectrometric (SOHO LASCO) C2 coronagraph at about 01:48 UT on 15 March. The arrival of the interplanetary shock, in the early hours of 17 March 2015, triggered a strong geomagnetic storm. The Dst (Disturbance Storm Time) index (Fig. 2C) increased up to about $+50 \mathrm{nT}$ at around 05:00 UT before decreasing sharply - first to a minimum of about $-73 \mathrm{nT}$ at 10:00 UT and then, after a short reversal, to an absolute minimum of about $-223 \mathrm{nT}$ at 23:00 UT. During the main phase of the storm, both, the local $K$ index measured at Dourbes (Fig. 2B) and the planetary $K_{\mathrm{p}}$ index, reached a maximum value of $7 \mathrm{KU}$. The storm started to slowly subside in the early hours of $18 \mathrm{March}$, and the field continued its recovery in the course of the following few days including the day of the eclipse. On the eclipse day, 20 March, Dst was still below the nominal storm-time value of $-50 \mathrm{nT}$ while the local $K$ index at Dourbes varied, for most of the day, between $K=3$ and $K=4$, an indication of "moderate" to "active" conditions. On the following day, 21 March, conditions were much closer to normal, with $K=2$ and Dst around $-35 \mathrm{nT}$ most of the time.
This geomagnetic storm was accompanied by an ionospheric storm as seen from the ionospheric measurements carried out at Dourbes. On the first day of the storm (17 March), the TEC (Fig. 2D) formed a two-peak pattern around noon, with the higher peak at almost 50 TECU, which was an increase of about $33 \%$ above the peak value on the previous day. The storm was at its full strength at midnight, 17-18 March, resulting in a substantial depletion of the ionosphere on the following day, 18 March, with peak TEC values below $20 \mathrm{TECU}$, a decrease of about $60 \%$ below the peak value at the beginning of the storm. On 19 March, one day before the eclipse, the TEC appeared to have recovered to almost pre-storm values during day time but remained significantly lower during night time. Similar to the TEC, the critical frequency $\left(f_{o} \mathrm{~F}_{2}\right)$ also went first through a "positive phase", followed by a negative phase lingering over the next couple of days (Fig. 2E). The night-time decrease was much more substantial, with $f_{o} \mathrm{~F}_{2}$ values dropping down to $1.5 \mathrm{MHz}$ which was barely above the minimum ionospheric frequency. It somewhat recovered during the night before the eclipse with $f_{o} \mathrm{~F}_{2}$ values of about $3.5-4.0 \mathrm{MHz}$. The diurnal variation of the F-layer peak height is normally in antiphase with that of the critical frequency. During the main phase of the storm, in the afternoon and evening hours of 17 March, the (virtual) peak height, $h^{\prime} \mathrm{F}_{2}$ (Fig. 2F), increased substantially and experienced large variability. The latter persisted well into the early hours of the following day and was still present in the following night. Afterwards it was back to its normal 
values. During the storm period, on and after 17 March, the values of the ionospheric slab thickness (Fig. 2G) were well above the medians, especially during night time, when the increase was regularly more than $100 \%$. This increase lasted even into the third day of the storm period (19 March) and it can be explained with the extremely low values of $f_{o} \mathrm{~F}_{2}$. By the time of the eclipse and the following day however, the slab thickness returned to normal again.

The selection of the control day was made in such a way as to ensure that the geospace conditions were normal (undisturbed) and that the control day was as close as possible to the day of the eclipse to avoid the effects of the annual variations. Naturally, the prime candidates for the control day were the day before the eclipse and the day after the eclipse. However, after taking into consideration the occurrence of the geomagnetic storm, it was decided that the day after the eclipse was more appropriate to serve as a reference. At that time, the storm was near the end of the recovery phase and the conditions were more or less back to normal.

\section{Observations during the eclipse}

Coronal observations were not carried out from the ground during the eclipse on 20 March 2015 because Brussels was outside of the eclipse totality band. Only a partial eclipse occurred which does not allow access to the solar corona. Also, the meteorological conditions on that day were unfavourable and clouds above Brussels obstructed the view during the partial eclipse. Only a few white-light images of the photosphere were obtained through a small break in the clouds that lasted for less than a minute. One of these images (Fig. 3) shows a largely spotless Sun, with only a few scattered pores. Nonetheless, a group of bright faculae was seen near the South-West limb. The $d y n$ method (Lemaire \& Stegen 2016) could have been used with 3D coronal density distributions like those currently derived from tomographic and stereoscopic observations of the corona. Unfortunately, the lack of coronal electron density distributions prevents us from obtaining the electron temperature distribution for this event.

As mentioned in the previous section, the USET context images and imagery from other telescopes indicate that the Sun only featured a large solitary H spot (NOAA AR 12303) in the North-East quadrant that was occulted during the eclipse, as seen from Brussels. This unipolar $\mathrm{H}$ spot, which marks the final decay stage of a past active region, did not evolve significantly during its entire disk transit. Such old spots are largely inactive. On the other hand, the dominant sunspot group of the preceding days (NOAA AR 12297) had just rotated beyond the West limb by the time of the eclipse, and is not visible any more, except for a few peripheral faculae at the limb. So, on the eclipse day, the Earth-facing hemisphere of the Sun did not feature any potential source of significant flaring activity.

On 20 March 2015, the PROBA2 spacecraft's orbit carried it through the darkest parts of the Moon's shadow two times first, between 08:28 UT and 08:53 UT and, again, between 10:24 UT and 10:50 UT.

SWAP observed both of them during an extended image acquisition sequence using a 1-min cadence. Because the spacecraft rotates $90^{\circ}$ approximately every $25 \mathrm{~min}$ to ensure its star trackers constantly have a view of the sky unobstructed by the Earth, some images were blurred by the spacecraft

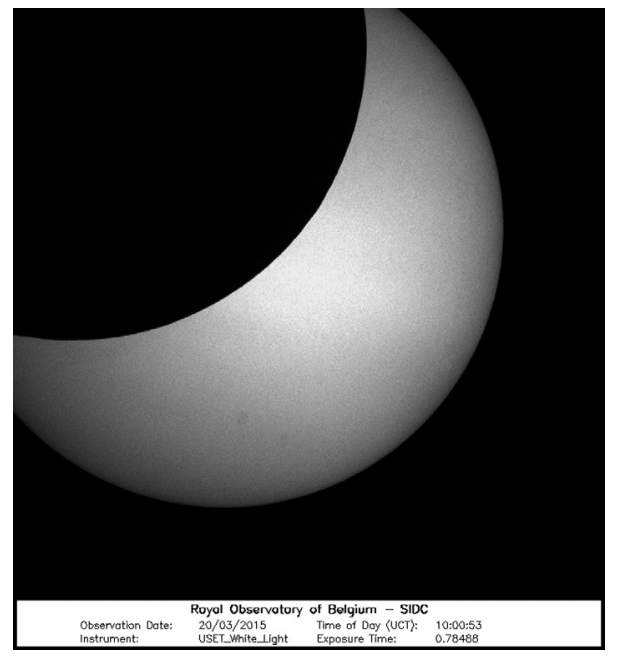

Fig. 3. USET white-light image taken at 10:00:53 UCT on 20 March 2015. Celestial North is in the vertical direction ( $P$ angle: $-25.1^{\circ}$; North solar pole tilted towards upper right). Except for a few faculae near the South-West limb and a couple of small pores in the North-West sector, the unobstructed part of the solar disk is almost featureless. Note: the fuzzy grey circles are artefacts due to dust specks.

motion and could not be recovered. However, the resulting sequence included high-quality images of both transits through the shadow and additional high-cadence images in a five-hour window that extended before and after the transits for context and in support of several special joint eclipse-spacecraft observing campaigns.

SWAP also carried out a sequence of off-pointing imaging beginning approximately $1 \mathrm{~h}$ before the eclipse and continuing until $1.5 \mathrm{~h}$ after the end of the eclipse. The resulting images were assembled into two high-dynamic-range, wide-field mosaic images, which reveal the EUV coronal structure to about two solar radii, beyond the nominal SWAP field of view. Because EUV images reveal only the corona at specific temperatures, comparing these mosaics to ground-based images of the eclipse in white light provides a useful diagnostic of the temperature structure of the extended corona at the time of the eclipse.

On 20 March 2015, a long duration C7.9 class flare from AR 12297 peaking at 01:33 UT was the only significant event of the day. This flare had an associated partial halo CME, emerging largely to the West of the Sun. During the eclipse (Fig. 4), the top of AR 12297 could be seen to the West of the occulting moon and AR 12305 to the East, which can be seen as a complex magnetic structure highlighted by the hot plasma trapped on the containing field lines. Two small prominences were evident to the North-East of the solar disk, but remained stable throughout the duration of the eclipse. The polar regions of the Sun are evident from the largely radial field structures, especially to the South where a large polar coronal hole was located.

LYRA eclipse data are typically used for analysing how the various regions of the solar disk participate in the EUV emission of the Sun. In particular, eclipse data help in validating limb darkening models (e.g. Shapiro et al. 2013). LYRA was operated with its high-quality backup channels during the entirety of the eclipse. During the first transit in the eclipse zone, the usual LYRA backup unit 3 was activated in parallel to the nominal unit 2. But during the second transit, 
(A)

(B)
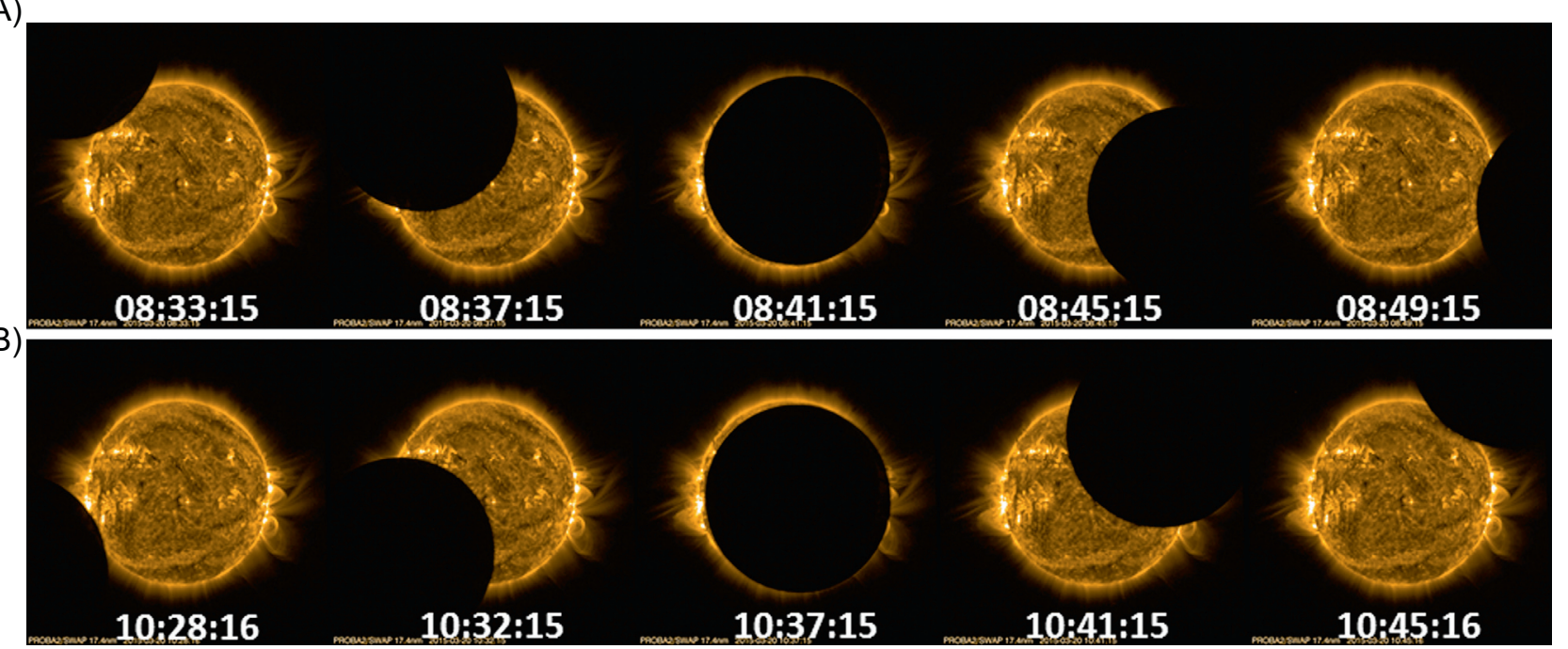

Fig. 4. SWAP images taken during the two passes through the Moon's shadow: 08:28-08:53 UT (A) and 10:24-10:50 UT (B).

(A)

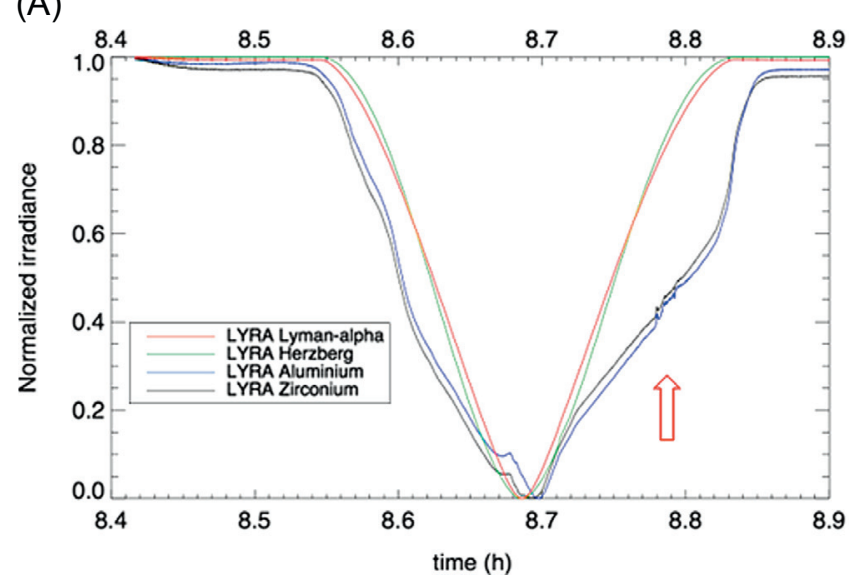

(B)

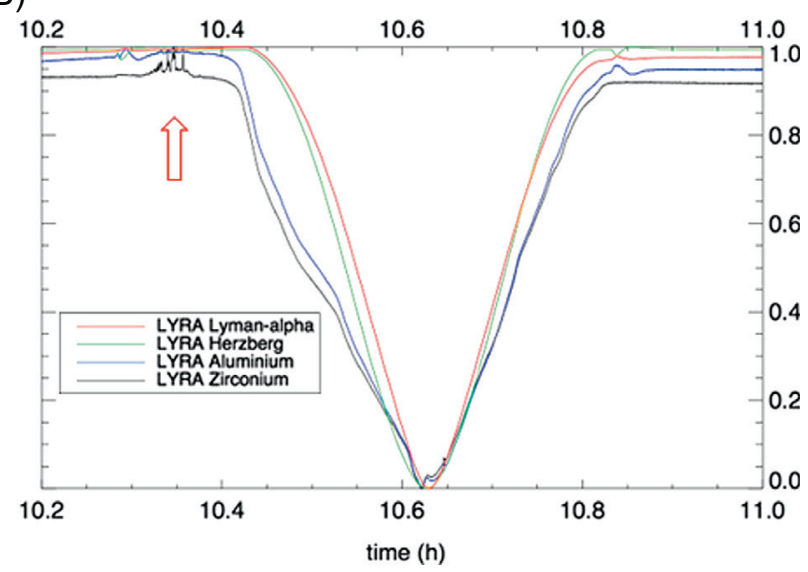

Fig. 5. LYRA transit measurements during the eclipse on 20 March 2015. Note the perturbations, indicated with red arrows, at around 08:47 UT (A) and 10:21 UT (B).

an exceptional campaign with the calibration unit 1 was set up (again in parallel to the nominal unit). This calibration unit is rarely used for observation campaigns to preserve it from degradation. Nevertheless, on this very occasion, we decided to use it to have a chance to capture the eclipse in the best observational conditions. The acquisition cadence was $20 \mathrm{~Hz}$ throughout the whole campaign. The two transits, respectively, observed by unit 3 and unit 1 , are plotted in Figure 5 . The shortest-wavelength channels (in black and blue colours), which are the most sensitive to solar activity, show slight distortions with respect to the very regular drop for the channels corresponding to wavelength ranges less affected by solar activity (red and green). These distortions correspond to the occultation of the bright limb active regions that are seen in SWAP images. Interestingly, small-amplitude perturbations are also visible in the black and blue curves around 08:47 UT and 10:21 UT. Those perturbations, which appear when PROBA2 is crossing the auroral regions, are due to the (decaying) geomagnetic storm.

The limb darkening, i.e. the density distribution across the solar disk, is a wavelength-dependent stellar feature which has important applications in the solar atmosphere modelling.
The mathematical relations describing this feature - the limb darkening functions (LDF) - have been studied by Neckel \& Labs (1994). They built an extensive database of LDF, in the 303-1099 nm wavelength range, by parameterising data obtained from a telescope at Kitt Peak National Solar Observatory. The SOLSPEC observations of the eclipse allow for comparison of the above-mentioned ground-based derived LDF with data from a satellite instrument providing a straightforward measurement of the limb darkening. Due to the constraints of the ISS orbits, the eclipse was only observed during its last phase, starting at an occultation of about $30 \%$. The decrease in the solar signal observed by SOLSPEC at $473 \mathrm{~nm}$ as a function of the normalised Sun to Moon distance, $|X|$, is shown in Figure 6 in comparison with the LDF function at $473 \mathrm{~nm}$. The ratio between SOLSPEC's observation and the LDF at $473 \mathrm{~nm}$ yields a good agreement, the deviation between them seldom exceeding 5\%. Thus, the SOLSPEC result can be considered as validation of the parameterised telescope data by space-based measurements.

Although the CALLISTO receiver monitors 200 frequencies between $270 \mathrm{MHz}$ and $870 \mathrm{MHz}$, only five frequencies spread over this frequency range will be presented. 


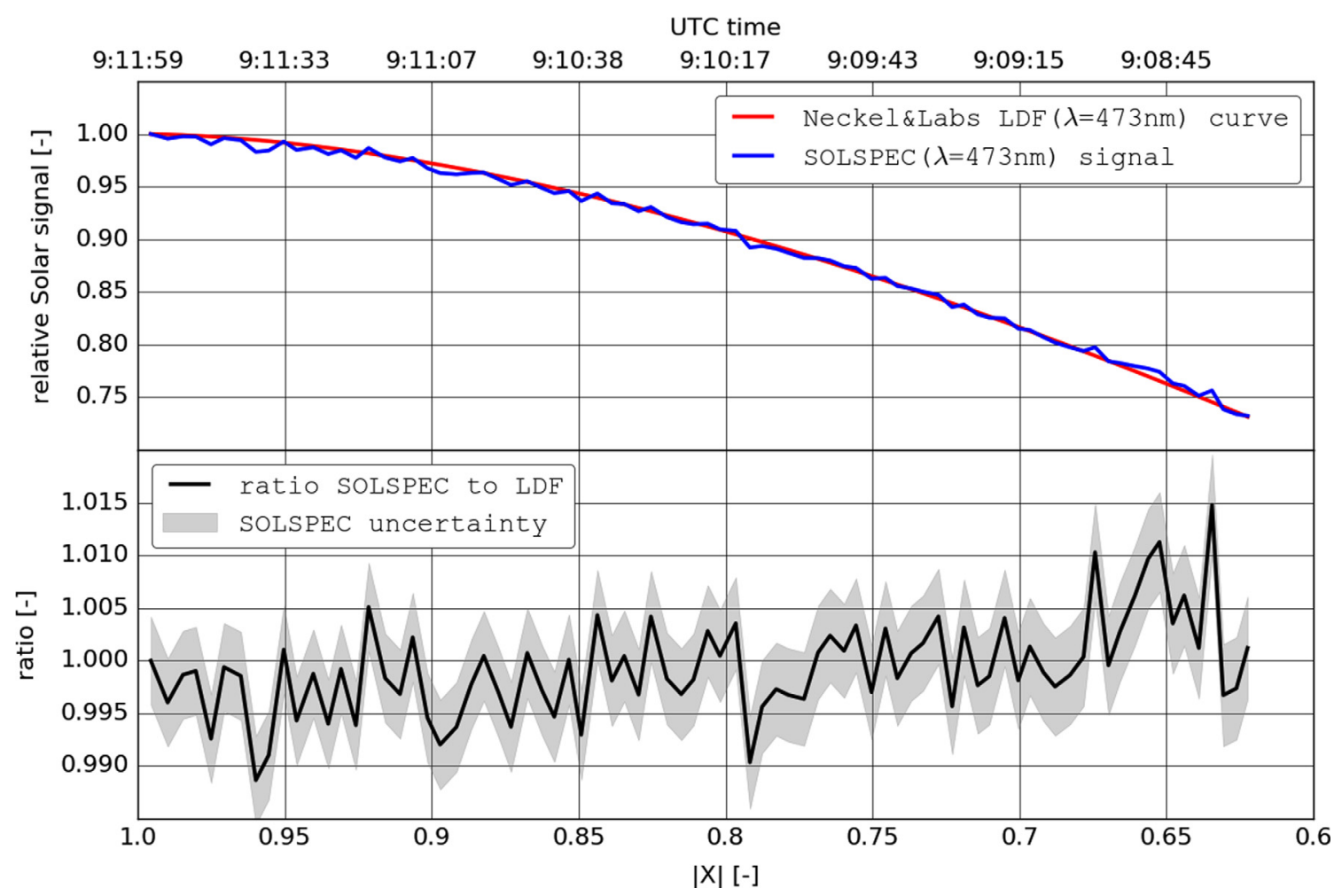

Fig. 6. Comparison between SOLSPEC's signal decrease due to solar occultation and the respective LDF. $|X|$ stands for the normalised distance between the Sun and the Moon centres. $|X|=1$ and $|X|=0$ correspond to the moments immediately after the fourth contact and before the first contact, respectively. SOLSPEC's Sun visibility window started at 09:08:28 UTC, corresponding to $|X|=0.62$.

(A)

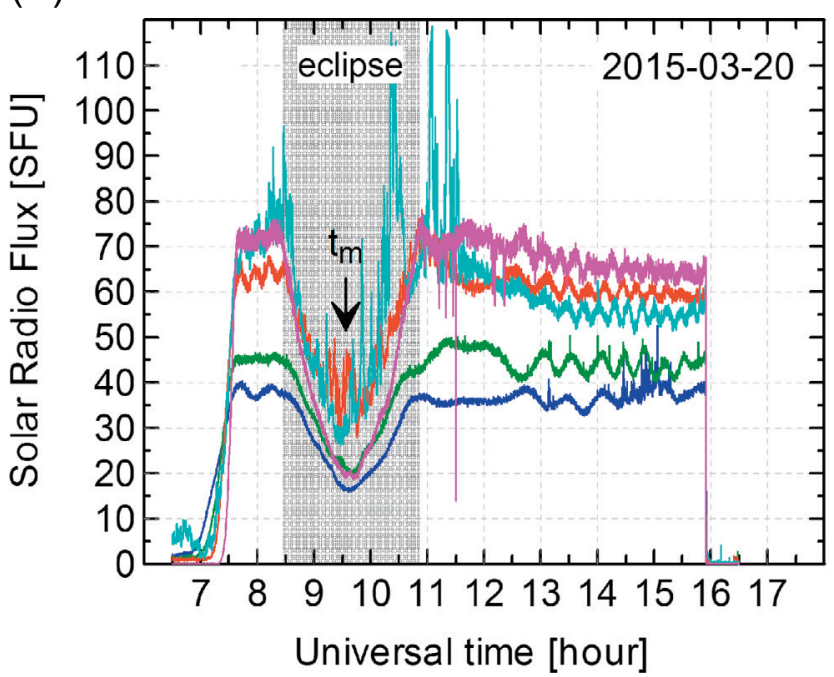

(B)

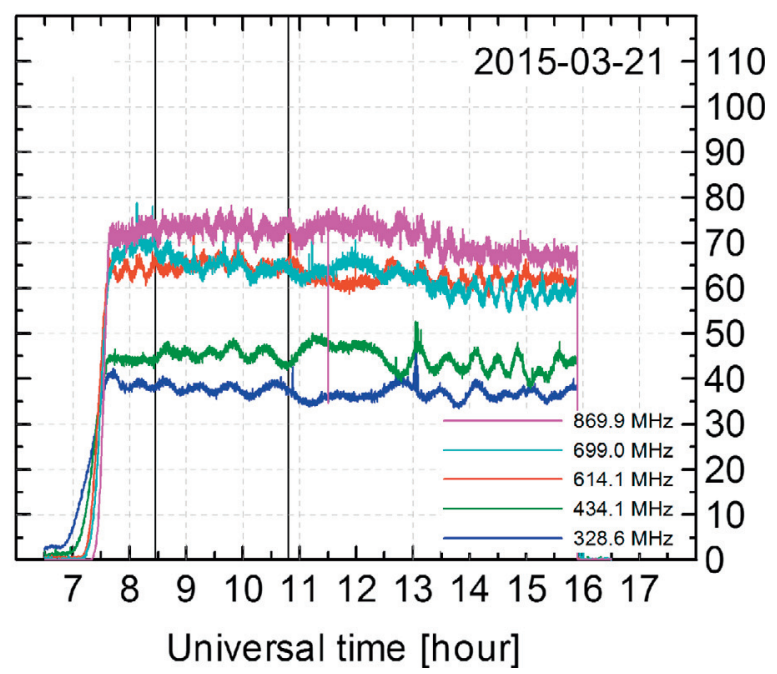

Fig. 7. Solar radio flux (in Solar Flux Units, $1 \mathrm{SFU}=10^{-22} \mathrm{~W} \mathrm{~m}^{-2} \mathrm{~Hz}^{-1}$ ) measured in Humain on the eclipse day, $20 \mathrm{March} 2015$ (A), and on the control day, 21 March 2015 (B), at five selected frequencies ranging from $328.6 \mathrm{MHz}$ to $869.9 \mathrm{MHz}$.

The different radio intensity curves for the eclipse and reference days (Fig. 7) show the rise of the Sun, around 07:30 UT (08:30 LT), while the solar radio telescope was pointing towards the East. It then started tracking the Sun until approximately 16:00 UT (17:00 LT). Spikes on the radio intensity curves are due to either solar bursts (if appearing on different curves at the same time) or interference; in this case, the latter. The ripples are caused by solar radiation reflected by the ground and interfering with the radiation "collected" directly by the telescope parabola.

During the eclipse (Fig. 7A), the flux decreased as the Moon passed in front of the Sun. The "radio" Sun is more extended than the optical one because the radio emission at the frequencies that are monitored originates from the upper layers of the solar atmosphere (corona). Therefore, even though the eclipse magnitude at Humain was 0.815 (Table 1) 
(A)

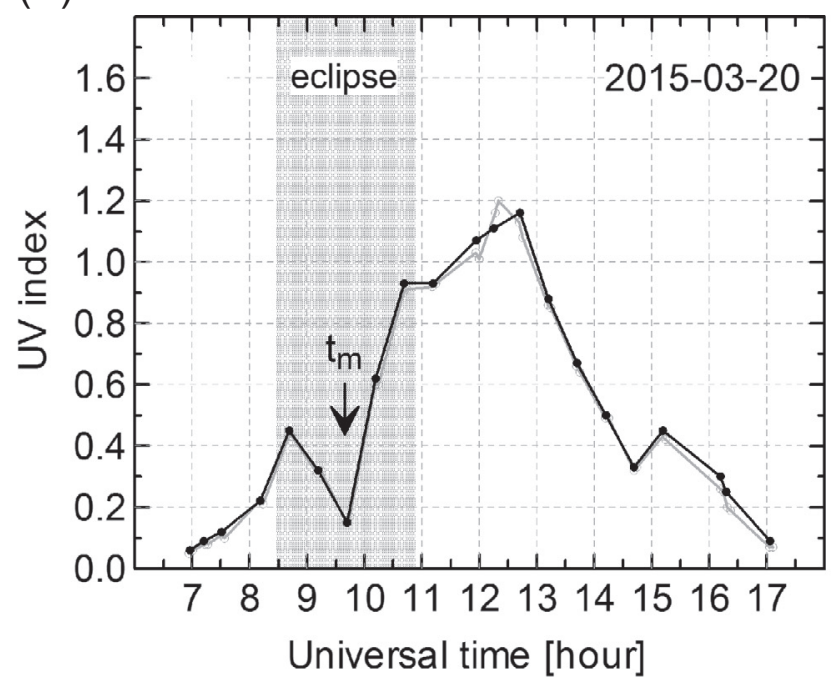

(B)

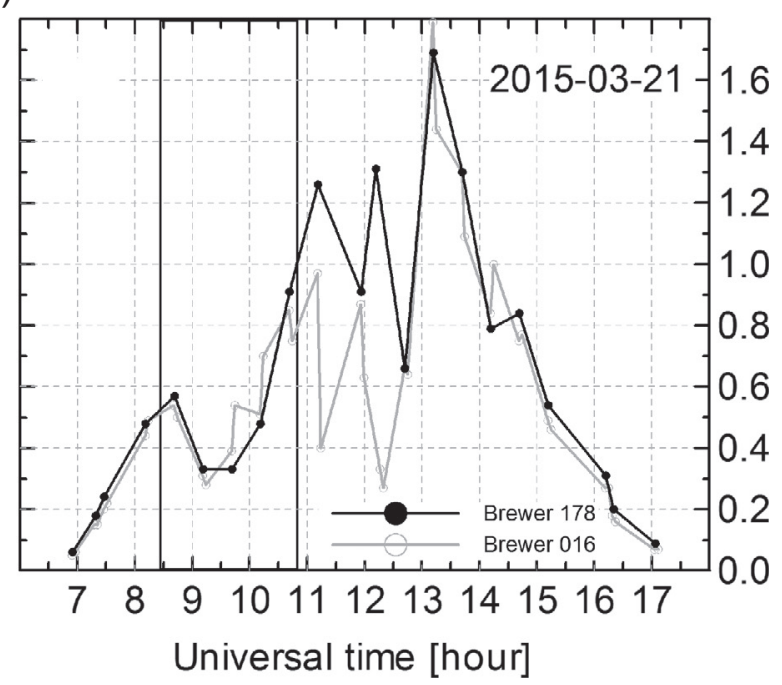

Fig. 8. Evolution of the effective UV index (based on Brewer spectrophotometric measurements) at Uccle (Brussels) during the eclipse day (A) and the control day (B).

in white light, the decrease of the radio flux at the maximum was only $55 \%, 56 \%$ and $74 \%$ at $328.6 \mathrm{MHz}, 434.1 \mathrm{MHz}$ and $869.9 \mathrm{MHz}$, respectively. Moreover, the corona is not uniform, due to the presence of active regions and other largescale coronal structures, which explains why the dip in the radio flux during the eclipse is not symmetric in its decreasing and increasing parts.

At Uccle (Brussels), spectral measurements (286-363 nm) in the UV are performed with Brewer spectrophotometers. From these measurements, the UV index is derived following the WMO (World Meteorological Organisation) definition. The results for the eclipse day (Fig. 8A) show a pronounced dip during the eclipse with the index falling down to 0.15 . Since there were clouds during the whole day, the UV index was below the calculated clear sky values. On the day after the eclipse (Fig. 8B), clouds were optically thicker, so the UV-index values were again low and varied widely.

The eclipse effects on the plasmasphere were expected to be rather limited, mainly due to the very low densities of both the neutral and ionised components. However, plasma transport to/from the plasmasphere should take place during eclipses. Unfortunately, on this occasion, we did not have direct plasmaspheric observations overhead. Even if we had, it would have been difficult to observe the effects of the eclipse since densities can only be measured along the spacecraft orbit. Instead, the three-dimensional coupled ionosphere-plasmasphere (3DPS) model (Pierrard \& Stegen 2008; Pierrard \& Voiculescu 2011) was used here to calculate the electron density profiles in both the ionosphere and the plasmasphere on 20 and 21 March 2015. The 3DPS model has been extensively tested and used on numerous occasions (see references above) proving it to be a reliable research tool. The model results presented here (Fig. 9) are for the location of Dourbes. At the time of the eclipse maximum, the electron density decreased slightly, contrary to what was observed on the day after. The density increased at 12:00 UT following the exposure of the atmosphere to the Sun. The solar eclipse resulted in a small reduction of the density, when compared to the profile at 8:00 UT, mainly in the ionosphere near the density peak height and in the lower plasmasphere. The position of the maximum density was slightly higher during the eclipse (at 09:30 UT) than before (at 08:00 UT). The position of the plasmapause, visible as the sharp decrease in density at large radial distances, varied during the eclipse but its variability was due to geomagnetic activity rather than to solar radiation.

The eclipse effects on the ionosphere will be presented first via the GNSS TEC measurements, then by vertical incidence sounding (VIS) measurements, and finally by combined GNSS and VIS measurements.

To investigate and demonstrate the TEC response to the eclipse on a regional scale, maps of the TEC difference between the eclipse and the reference day have been produced. The relative difference, $\mathrm{TEC}_{\mathrm{rel}}=\left(\mathrm{TEC}_{\mathrm{ecl}}-\mathrm{TEC}_{\mathrm{ref}}\right) / \mathrm{TEC}_{\mathrm{ref}}$, is calculated on each grid point from the corresponding TEC values on the eclipse day $\left(\mathrm{TEC}_{\mathrm{ecl}}\right)$ and the reference day $\left(\mathrm{TEC}_{\mathrm{ref}}\right)$. Several "difference" maps (relative percentage deviation), together with the corresponding TEC maps from the eclipse and reference days, are displayed here (Fig. 10) at different stages during the eclipse.

The TEC maps from the reference day (Fig. 10B) show the regular morning TEC increase appearing first in the South-East (SE) corner and progressing steadily towards the West (W). As a result, the early morning TEC values in the North-West (NW) corner also increase steadily from about 6-7 TECU (at 07:40 UT) up to about 14-15 TECU (at 11:50 UT). The corresponding TEC maps from the eclipse day (Fig. 10A) show that the TEC increase in the South-East is substantially lower and its progress towards the West is suppressed by the eclipse. In the North-West corner (along/near the total eclipse path), the TEC remained more or less unchanged (in the order of 6-8 TECU) until about 10:00 UT when it started to slowly increase and recover from the eclipse. In fact, the area of these relatively low TEC values increases towards the East as seen on the maps from 08:40 UT to 10:30 UT.

To further clarify the TEC behaviour let us examine the difference maps (Fig. 10C). The first map, at the nominal start of the eclipse at sea level (07:40 UT), shows that the eclipse-day TEC is about $10-20 \%$ different from the reference-day TEC. While the values are almost identical over large areas in the North-East (NE) and North-West (NW), 
(A)

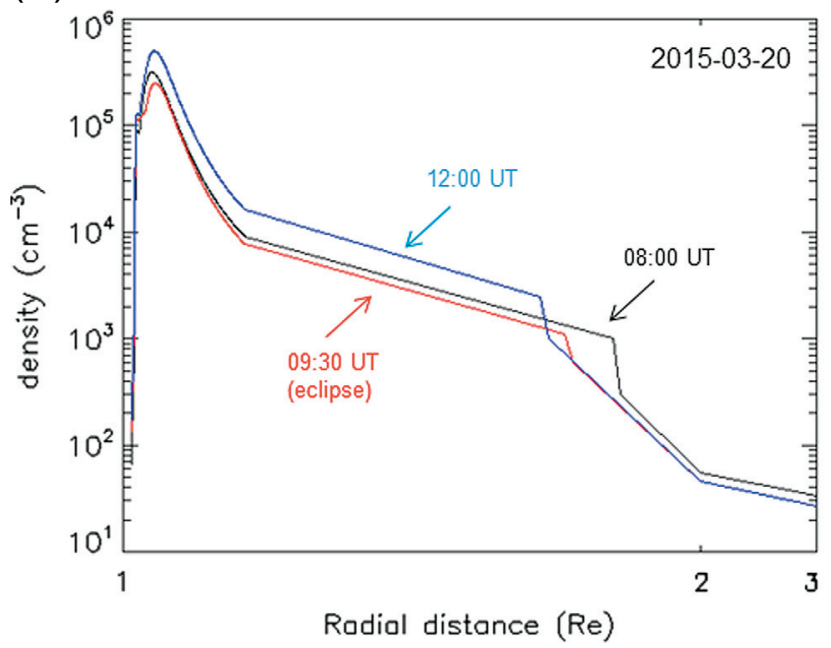

(B)

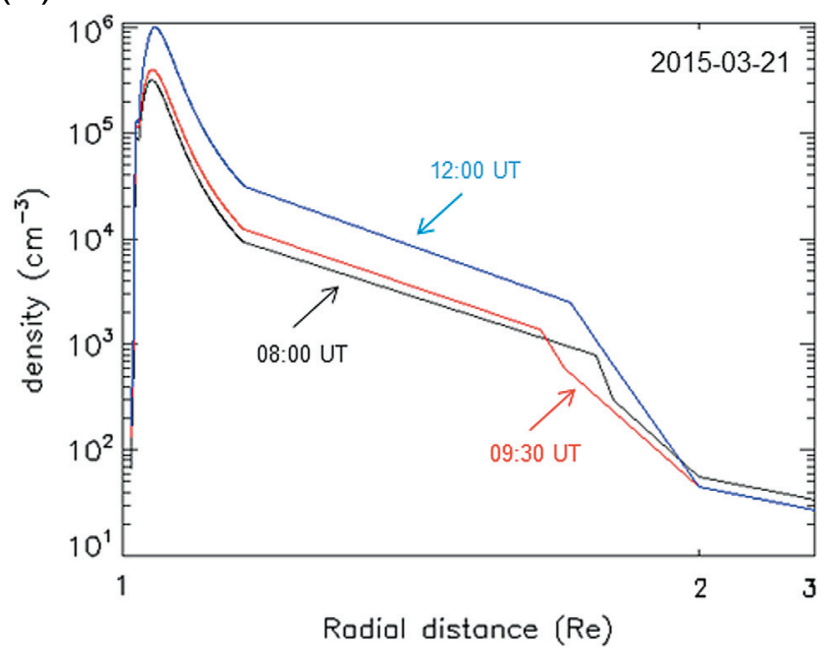

Fig. 9. Ionosphere-plasmasphere model simulations for Dourbes before the eclipse, 08:00 UT (black), at the eclipse maximum, 09:30 UT (red), and after the eclipse, 12:00 UT (blue). (A) Electron density profiles on the eclipse day, 20 March 2015. (B) Electron density profiles on the reference day, 21 March 2015.

$\mathrm{TEC}_{\mathrm{ecl}}$ is about $10-20 \%$ lower than $\mathrm{TEC}_{\text {ref }}$ over most of Europe. These relatively lower values can be attributed to the effects of the geomagnetic storm from a few days earlier, normal day-to-day variability and inaccuracies in the TEC mapping. However, the existence of a patch of larger negative deviations over Italy, stretching in South (S)-South-West (SW) direction (i.e. towards the location of the first contact of the penumbra in SW), suggests that its existence might also be an early eclipse effect. The latter is supported by the following map, at 08:40 UT, where the above-mentioned area of negative difference (depression) is shown significantly expanded towards SW reaching a minimum of about $-35 \%$ at locations around $35^{\circ} \mathrm{N}, 10^{\circ} \mathrm{W}$. Overall, the depression area of $\mathrm{TEC}_{\text {rel }}$ values below $-30 \%$, in the shape of an oval (in blue colour), appears to track the eclipse passage from South-West (08:40 UT), through West (the 09:10 UT map), to North-West (the 09:35 UT and 10:00 UT maps). On the map from 10:30 UT, the depression area is seen reduced with a minimum over Central Europe of about $-50 \%$. The last map, at the nominal end of the eclipse at sea level (11:50 UT), shows the depression area further reduced and confined to latitudes below $55^{\circ} \mathrm{N}$ with a minimum of about $-40 \%$ at locations around $48^{\circ} \mathrm{N}, 16^{\circ} \mathrm{E}$. The difference maps show that different locations experienced different levels of TEC depletion and that the depletion occurred at different times. A question rises of what the maximum depletion of TEC was and where did it occur during the eclipse. For this purpose, all $\mathrm{TEC}_{\mathrm{rel}}$ maps within the time interval from 07:40 UT to 11:50 UT were processed and the minimum $\mathrm{TEC}_{\text {rel }}$ value picked up for each grid point. The resulting map is shown in Figure 11A. Note that the map shows what the minimum was at each grid point but not the time at which it occurred. It is obvious that the depletion was more than $20 \%$ everywhere in Europe but the area of depletion more than $40 \%$ is a strip stretching diagonally in the NW-SE direction from the area of the total eclipse down to the area of $50 \%$ obscuration. It is interesting to note that maximum depletion of above $53 \%$ (i.e. minimum $\mathrm{TEC}_{\text {rel }}<-53 \%$ ) was reached on a patch over central Europe, where the maximum obscuration was between $70 \%$ and $80 \%$ rather than in the area of complete obscuration. This can be attributed to effects of the eclipse geometry which will be discussed in one of the following sections.

The next question is when did the maximum depletion occur with respect to the maximum eclipse at each location. For this purpose, at each grid point, we subtracted the time of the maximum eclipse from the time of the maximum TEC $_{\text {rel }}$ depletion, again within the time interval from 07:40 UT to 11:50 UT. The resulting map is shown in Figure 11B. Without exception, the difference is non-negative, meaning that during the eclipse the TEC depletion is either instant or delayed. An area where the reaction was instant is on the path of totality near the point of the greatest eclipse. Other areas of immediate response are the South-West and the North-East. There is an area of moderate delay, between 10 and $30 \mathrm{~min}$, stretching all the way from the North-West $(100 \%$ obscuration $)$ to the South-East (20\% obscuration). The areas located in-between the "instant-response" and "moderate-response" areas are characterised with substantial delays of more than $30 \mathrm{~min}$, reaching local maxima of above $45-50 \mathrm{~min}$. It is difficult to explain this distribution of the delay. However, by comparing the "delay" map (Fig. 11B) with the maximal depletion map (Fig. 11A), it appears that, in general, the largest delay is observed in the areas of the largest gradient in the maximum TEC depletion.

Vertical TEC (vTEC) time series (cf. Fig. 12) were extracted from the ROB-IONO maps at three locations experiencing different eclipse magnitudes: one location affected by the total eclipse - the Faroe Islands $\left(62.0^{\circ} \mathrm{N}\right.$, $393.2^{\circ} \mathrm{E}$ ) two locations affected by the partial eclipse Brussels $\left(50.9^{\circ} \mathrm{N}, 4.4^{\circ} \mathrm{E}\right)$ with $\sim 80 \%$ obscuration and Belgrade $\left(44.5^{\circ} \mathrm{N}, 20.5^{\circ} \mathrm{E}\right)$ with $\sim 50 \%$ obscuration.

As indicated by the TEC values and their medians on the eclipse day (Figs. 12A, 12D and 12G), the ion production due to photoionisation was slower and substantially reduced. The reduction appears to be larger at lower latitudes (Belgrade). However, the reduction during the eclipse from both, the median and the reference-day values, is much larger at the Faroe Islands, i.e. closer to the totality path. The datasets for the eclipse day (20 March) and the reference day (21 March) were compared with each other. Normally, the 
(A)

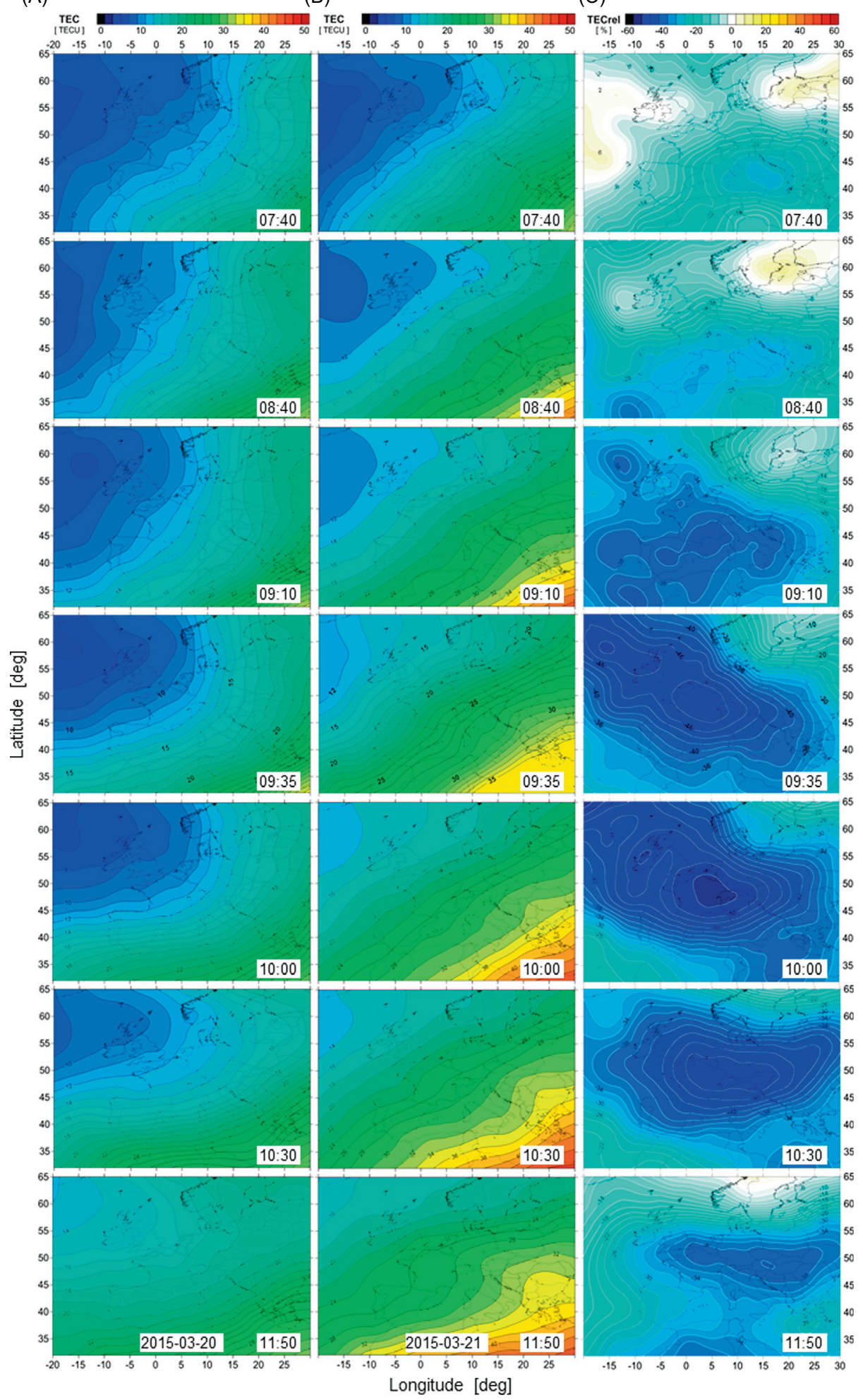

Fig. 10. European ROB-IONO TEC maps during the eclipse (A), at the same time on the control day (B) and the corresponding relative differences $(\mathrm{C})$.

TEC daily pattern should be similar over these two consecutive days, given that the solar radio flux index F10.7 remained almost the same (112.7 SFU and 113.7 SFU, respectively). The largest TEC differences between these two days were observed shortly after the maximum of the eclipse. In comparison with the reference day, during the eclipse the TEC values decreased much more at lower latitudes (up to 14-15 TECU)
(Figs. $12 \mathrm{G}$ and $12 \mathrm{H}$ ) but, in relative terms, the decrease was larger $(45-50 \%)$ at higher latitudes (Figs. 12C, 12F and 12I). The recovery was much quicker at higher latitudes despite the larger obscuration of the Sun. Also, the maximal difference occurred after the time of maximum obscuration $\left(t_{\mathrm{m}}\right)$. Such delayed response to the obscuration can be explained with the (diffusive and convective) transport processes dominating 

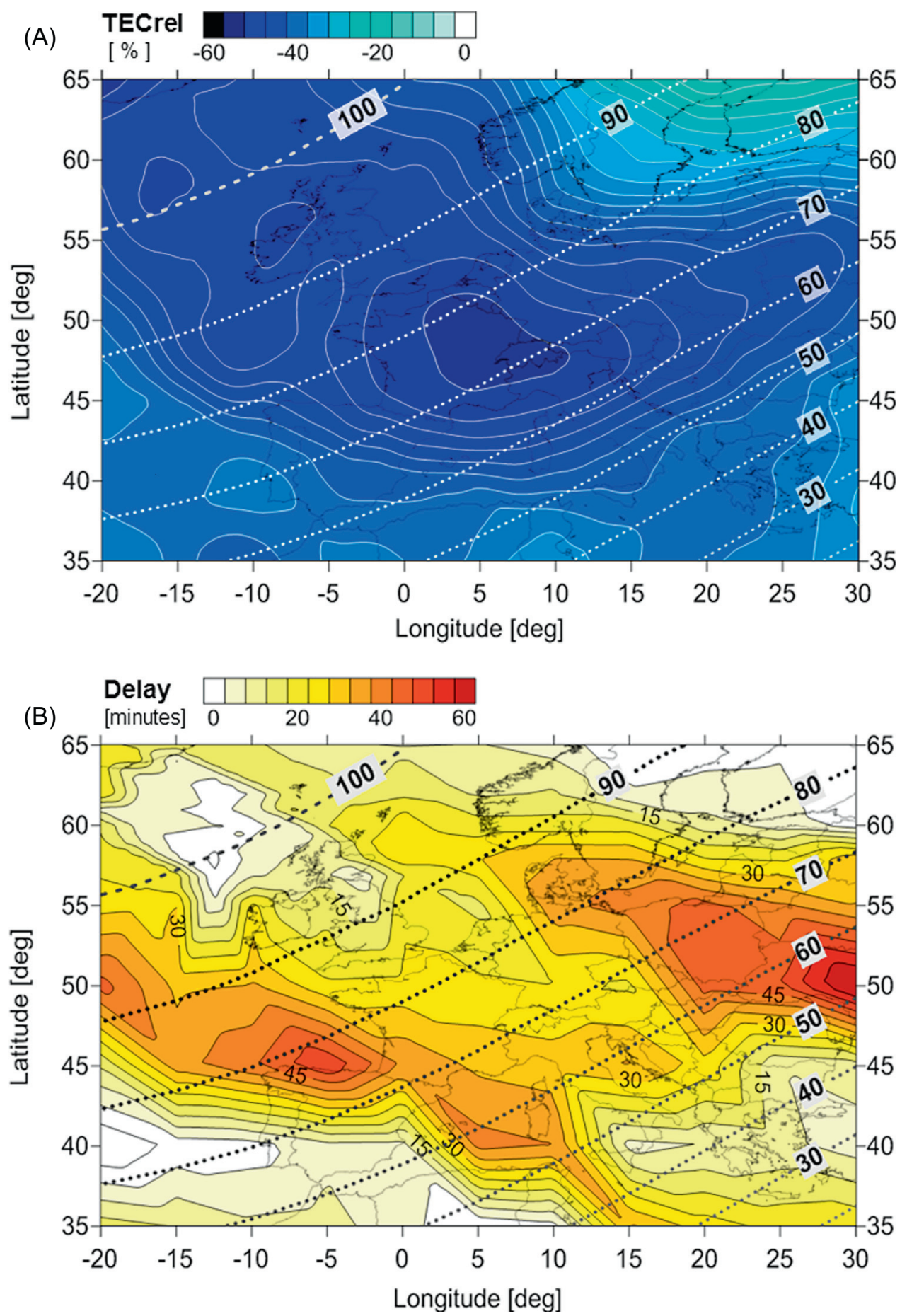

Fig. 11. (A) Estimation of the maximum TEC depletion based on the European TEC maps during the eclipse, compared with the obscuration (dashed lines) map. (B) Estimation of the TEC depletion delay based on the European TEC maps during the eclipse, comparison with the obscuration map.

the behaviour of the topside ionosphere and the plasmasphere. Therefore, the delay is expected to increase with altitude. Another explanation for the delay is in the eclipse geometry.

Local ionospheric measurements carried out at Dourbes are shown in Figure 13 for the eclipse day (Figs. 13A, 13C, $13 \mathrm{E}$ and $13 \mathrm{G}$ ) and the reference day (Figs. 13B, 13D, 13F and $13 \mathrm{H})$. Most instantaneous measurements are compared with the corresponding 27-day medians obtained from 4 to 30 March 2017.
On the reference day (Fig. 13B), the TEC increased steadily in the morning from around 10 TECU at 06:00 UT up to 26-28 TECU at 12:00 UT, which was to be expected for these hours of the day under normal conditions. Shortly after 12:00 UT, the TEC started to decrease and by 18:00 UT the values dropped to around 15 TECU. While the TEC daily pattern was typical for the season, all the instantaneous TEC values remained below the median values. Such behaviour is typical for the ionosphere when in a state of recovery after 

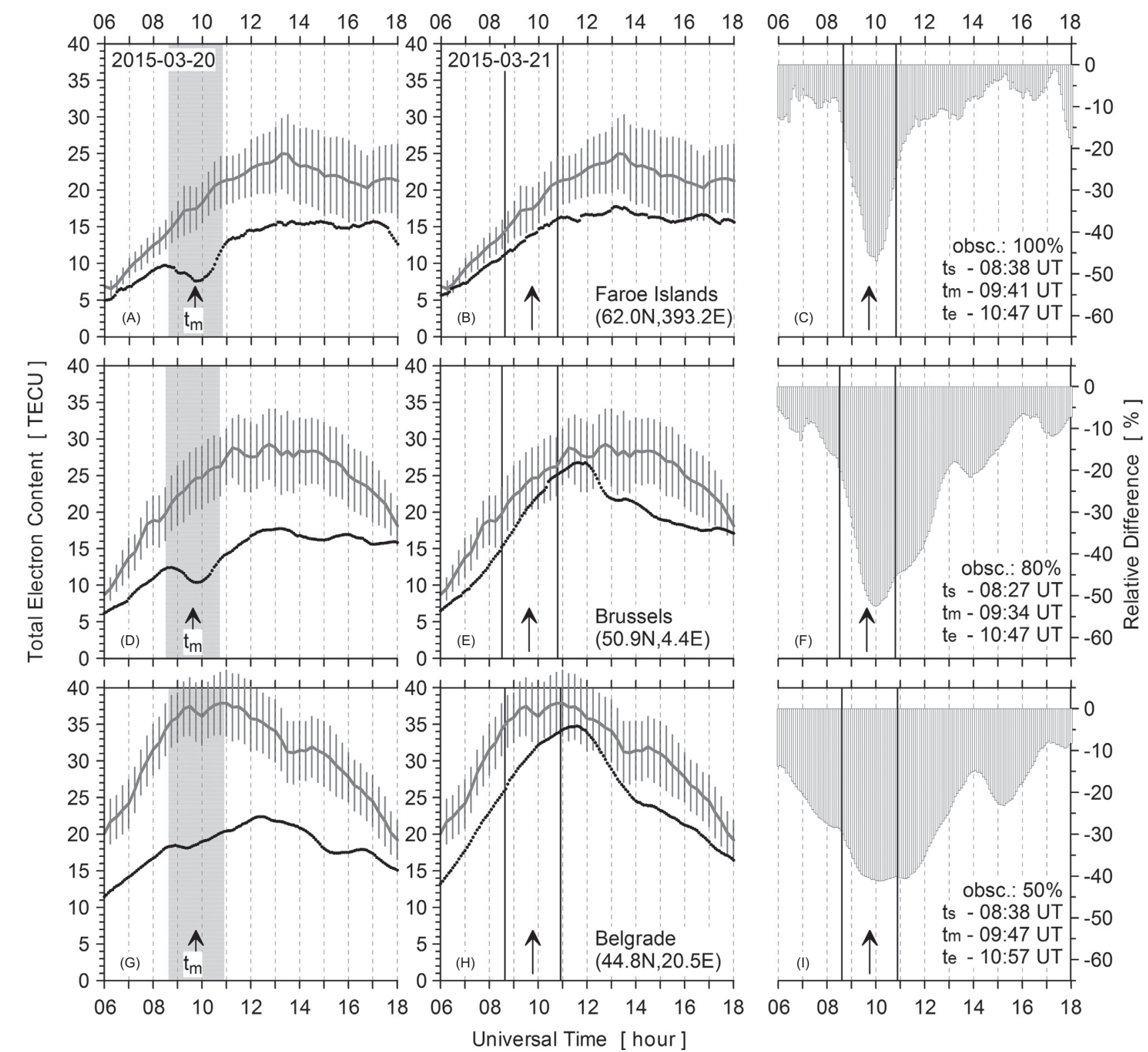

Fig. 12. Vertical TEC time series at three locations with different eclipse magnitudes: Faroe Islands (total, 100\% obscuration), Brussels (partial, 80\% obscuration) and Belgrade (partial, 50\% obscuration) for the eclipse day, 20 March 2015 (A, D, G) and the reference day, 21 March 2015 (B, E, H). The solid black lines indicate the instantaneous TEC measurements while the solid grey lines are the median TEC values based on the previous 15 days. The vertical bars show the standard deviations $(2 \sigma)$ centred on the median values. The shading indicates the eclipse period with the exact time of the maximum obscuration marked by an arrow. $(\mathrm{C}, \mathrm{F}, \mathrm{I})$ The relative deviations of the eclipse day measurements from the reference-day measurements.

a geomagnetic storm. On the day of the eclipse (Fig. 13A), the TEC behaviour was quite different. In the early morning hours, from 06:00 UT to shortly before 08:00 UT, the TEC followed the same trend as on the reference day, i.e. increasing from about 7-10 TECU to about 12-14 TECU. At around 07:41 UT the TEC values were consistently at a maximum of about 14.5 TECU and from then on they started to deviate from the reference, stagnating around 12 TECU before starting to decrease again at the time when the eclipse reached its maximum. The downward trend was preserved for some time after the eclipse maximum. TEC was at its minimum of 7.7 TECU at 09:58 UT and once again (7.8 TECU) half an hour later (10:27 UT). Thus, the net TEC decrease due to the eclipse was estimated at about 47\%. After its minimum at 10:27 UT, TEC started to increase (even before the end of the eclipse) and reached a daytime maximum of 22.5 TECU at 13:55 UT. The TEC values remained depressed, way below the reference-day values, throughout the afternoon, 12:00 UT-16:00 UT. After 16:00 UT, the TEC values were similar to those on the reference day.

Presented also are the variations of other key ionospheric characteristics - the critical frequencies of the $F$ layer $\left(f_{o} \mathrm{~F}_{1}\right.$ and $\left.f_{o} \mathrm{~F}_{2}\right)$ and the $\mathrm{E}$ layer $\left(f_{o} \mathrm{E}\right)$ together with the peak (virtual) heights of the $\mathrm{F}$ layer $\left(h^{\prime} \mathrm{F}_{2}\right)$ and the $\mathrm{E}$ layer $\left(h^{\prime} \mathrm{E}\right)-$ for both, the day of the eclipse (Figs. 13C and E) and the reference (Figs. 13D and 13F) day. These characteristics were manually scaled from the ionograms. During the eclipse, the deviation of the critical frequencies from their normal (quiet-time) diurnal behaviour is obvious. Normally, in the morning hours, the critical frequencies start to increase with 

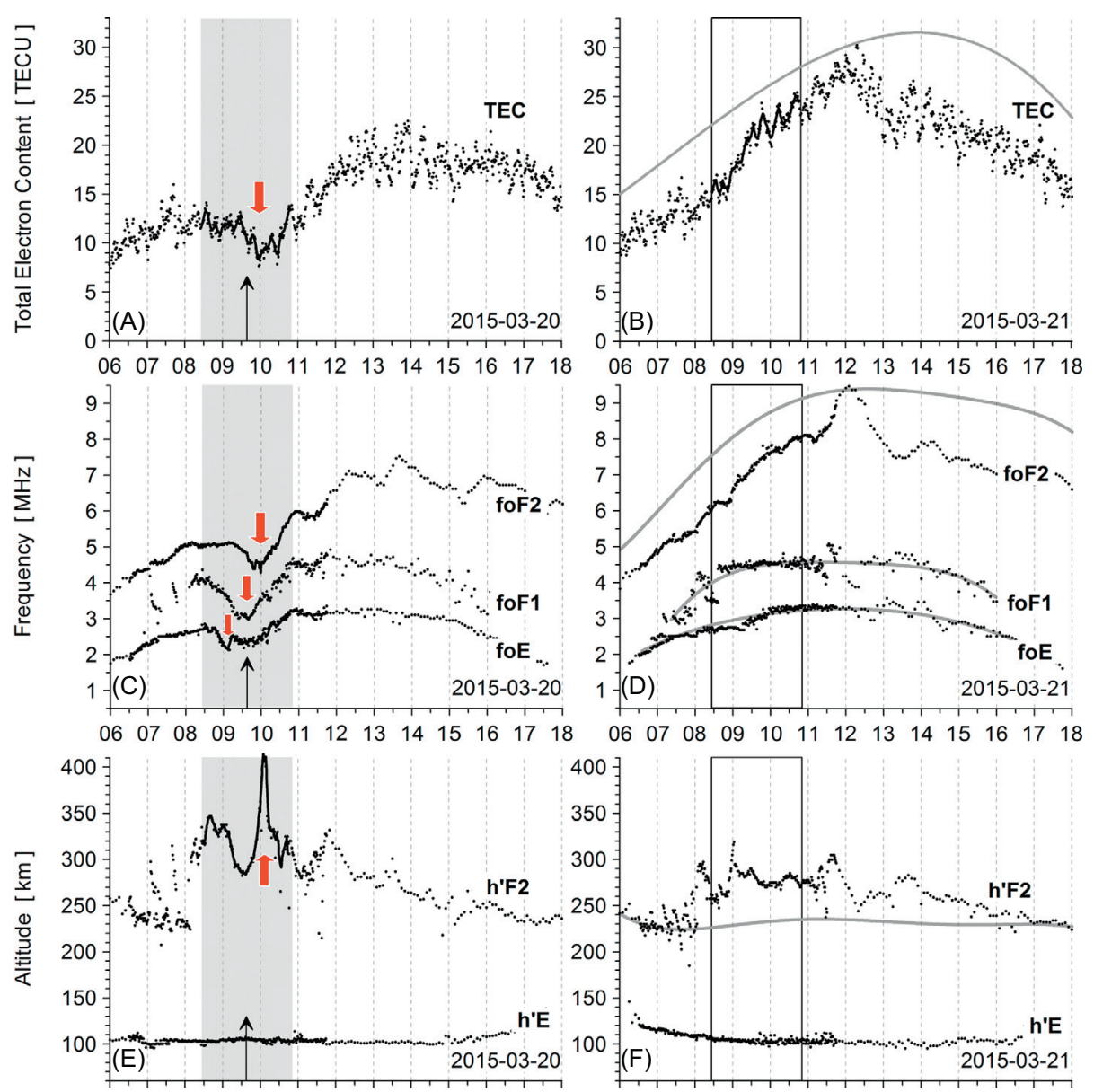

$\begin{array}{llllllllllllllllllllllllll}06 & 07 & 08 & 09 & 10 & 11 & 12 & 13 & 14 & 15 & 16 & 17 & 18 & 06 & 07 & 08 & 09 & 10 & 11 & 12 & 13 & 14 & 15 & 16 & 17 & 18\end{array}$

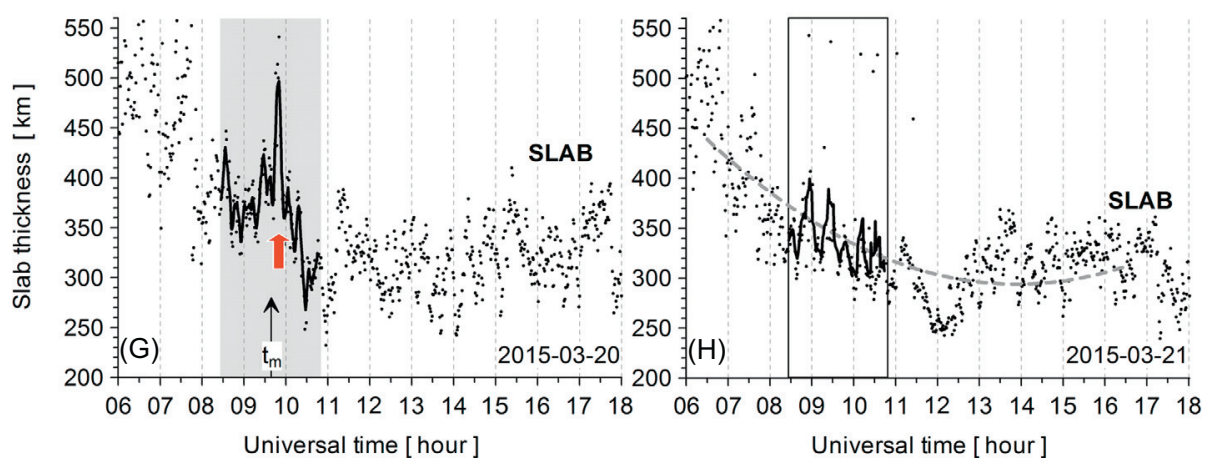

Fig. 13. Key ionospheric characteristics measured at Dourbes during the eclipse day, 20 March 2015 (A, C, E, G) and the reference day, 21 March 2015 (B, D, F, H). GPS TEC (A, B); $f_{o} \mathrm{~F}_{2}, f_{o} \mathrm{~F}_{1}$ and $f_{o} \mathrm{E}(\mathrm{C}$ and $\mathrm{D}) ; h^{\prime} \mathrm{F}_{2}$ and $h^{\prime} \mathrm{E}(\mathrm{E}$ and $\mathrm{F})$ and slab thickness $(\mathrm{G}$ and $\mathrm{H})$. Instantaneous measurements denoted with dots, the 27-day medians (based on data from 4 to 30 March) plotted with solid (grey) curves, and the (approximated) daytime trends are plotted with dashed lines. The time period of the eclipse is shaded and all measurements during that time frame are highlighted with solid black curves (7-point running average). Red arrows indicate the maximum deviation (from medians) during the eclipse.

the sunrise. On the day of the eclipse, however, this morningtime increase was interrupted by the eclipse. Shortly after the start of the eclipse, a pronounced decrease was observed in the critical frequencies. The $f_{o} \mathrm{E}$ minimum was reached first (about $30 \mathrm{~min}$ before the time of the maximum eclipse, $t_{\mathrm{m}}$ ), followed by the $f_{o} \mathrm{~F}_{1}$ minimum (at around $t_{\mathrm{m}}$ ), and then the $f_{o} \mathrm{~F}_{2}$ minimum (about $25 \mathrm{~min}$ after $t_{\mathrm{m}}$ ). After reaching the minima, the frequencies began increasing back towards their corresponding regular-day values. Opposite to the critical frequencies, the (virtual) height $h^{\prime} \mathrm{F}_{2}$ increased during the eclipse, peaking about $30 \mathrm{~min}$ after $t_{\mathrm{m}}$. Apparently, $h^{\prime} \mathrm{E}$ was unaffected by the eclipse.
The equivalent slab thickness (Fig. 13G), derived with high-time resolution, provided a detailed outlook on the shape variations of the vertical electron density profile during the eclipse. The calculations show that, indeed, the slab thickness increased substantially during the eclipse peaking at almost $540 \mathrm{~km}$ shortly after the maximum obscuration of the Sun an increase of more than $50 \%$ above the diurnal trend. This means that, taking into account the TEC and $f_{o} \mathrm{~F}_{2}$ measurements shown in the previous paragraphs, the vertical electron density profile is much "slimmer" but steeper, i.e. the ionosphere is depleted but extended in vertical direction. After the peak increase, the slab thickness started to decrease rather 

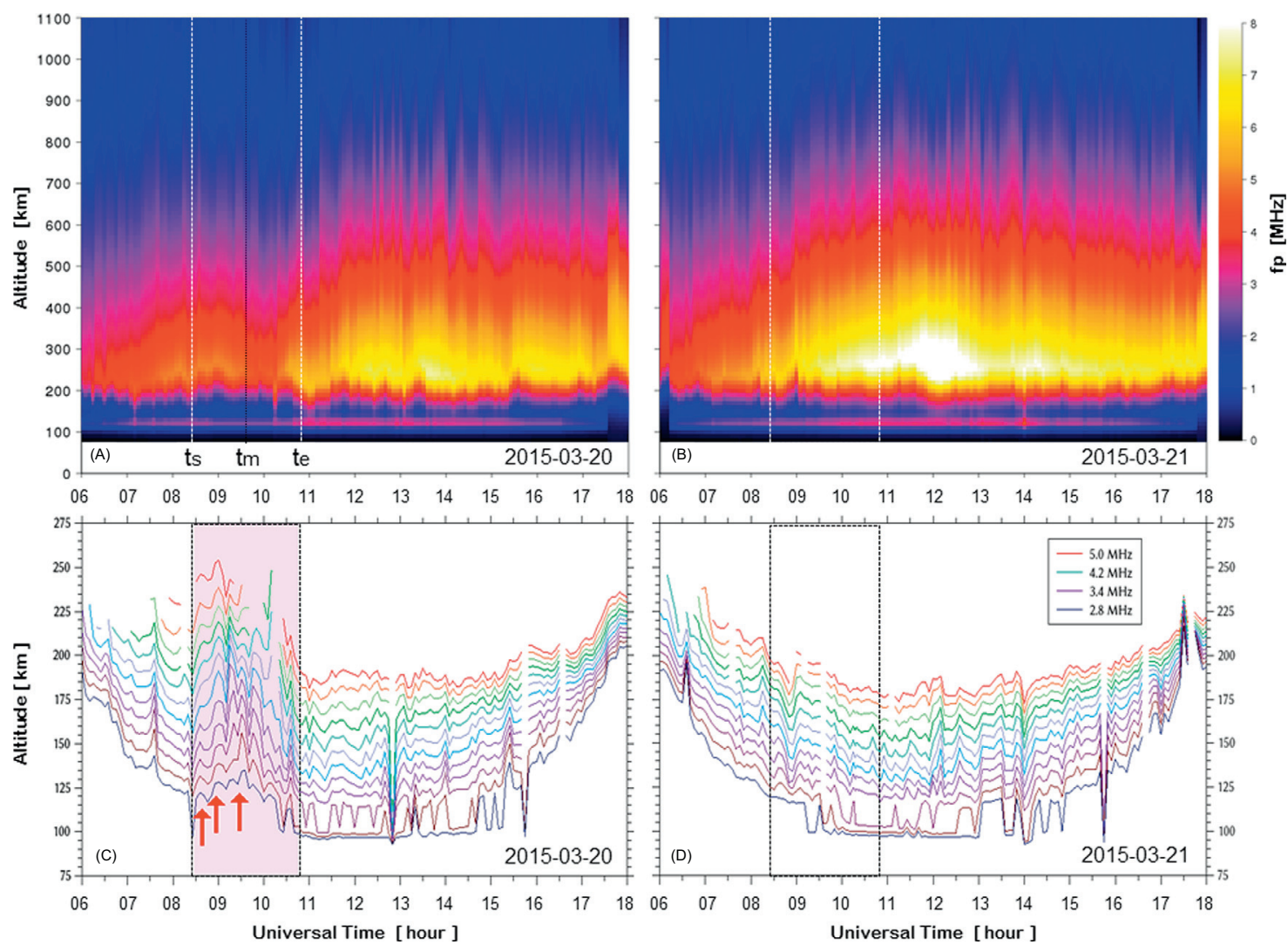

Fig. 14. Vertical plasma density distribution (converted to plasma frequency) over Dourbes, as reconstructed by the LIEDR system (5-min resolution), during the eclipse day (A) and the control day (B). True-height contours for frequencies ranging from $2.8 \mathrm{MHz}$ (bottom curve) up to $5.0 \mathrm{MHz}$ (top curve) in steps of $0.2 \mathrm{MHz}$ as obtained from Digisonde measurements at Dourbes during the eclipse day (C) and the control day (D). The shading indicates the eclipse time period, red arrows point at TID effects.

rapidly, even falling well below the trend by about $20 \%$. This decrease can be attributed to the eclipse-generated cooling of the thermosphere. In contrast, during the same period on the control day (Fig. 13H), the slab thickness values vary around the diurnal trend values by no more than $10 \%$, well within the standard variability of the slab thickness under normal daytime conditions.

Clearly, an eclipse affects all ionospheric layers, albeit to a different extent and in a different manner. At the bottom of the ionosphere, in the $\mathrm{D}$ and $\mathrm{E}$ layers, the amount of ionisation is mainly governed by local production and loss processes, while higher up, in the F region, the plasma transport processes play a much more pronounced role. The dynamic plasma redistribution processes that take place during eclipses lead to modifications in the shape of the vertical electron density profile in the ionosphere. Most notably, the electron density decreases in both the $\mathrm{E}$ and $\mathrm{F}$ layers of the ionosphere, accompanied by a simultaneous increase in the height of the $\mathrm{F}$ layer. In relative terms, the depletion of ionisation is more substantial in the lower ionosphere where photoionisation and recombination processes are more significant. Enhancement in the peak electron density height was to be expected; however, the presence of dynamic forces such as electric fields and (meridional) thermospheric winds may have complicated the picture and the height showed large variability during the eclipse.

For eclipse observations to be more efficiently used in ionospheric studies, detailed electron density profiling is a necessity. For this purpose, we provide next the LIEDRreconstructed vertical distribution of the ionospheric plasma density (presented in terms of plasma frequency) above Dourbes (Figs. 14A and 14B). The resolution is $5 \mathrm{~min}$ in time and $5 \mathrm{~km}$ in altitude. The local ionospheric parameters, scaled automatically from the Digisonde ionograms, together with the vertical TEC values calculated from the GNSS measurements, are used as input for the LIEDR system. The results show that, during both days, the ionosphere was still recovering from the ionospheric storm that started on 17 March 2015, with plasma density still below the median. Nevertheless, during the eclipse (Fig. 14A), a pronounced decrease in the ionisation level is visible as a result of the decrease in the production rate. It should be noted that an eclipse event happens rather quickly compared to many ionospheric processes, and that the ionosphere does not get sufficient time to reach equilibrium before the eclipse has passed and the production starts to increase again. The depletion in the topside ionosphere is clearly visible and is strongest between $t_{\mathrm{m}}$ (maximum eclipse) and $t_{\mathrm{e}}$ (end of the eclipse). Obviously, there is a delay in the ionospheric reaction which increases with altitude, confirming the notes made with regard to the observations presented in the previous figures.

During eclipses, the occurrence of various wave phenomena, such as atmospheric gravity waves (AGWs) and travelling ionospheric disturbances (TIDs), is to be expected. Gravity waves are known for contributing to the atmospheric dynamics, from the Earth's surface up to the mesosphere and lower thermosphere. Properties of the "neutral" atmospheric waves, such as period and wavelength, are closely reflected by the electron density fluctuation due to the coupling of the neutral and ionised components of the atmosphere. Once coupled, 
(A)

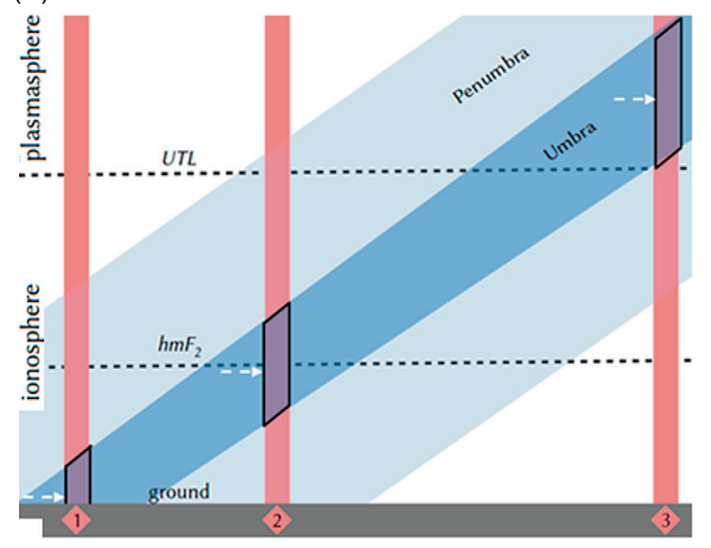

(B)

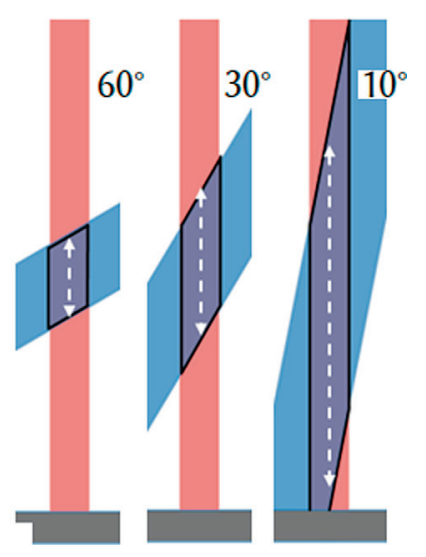

Fig. 15. Eclipse geometry (Earth's curvature ignored) with respect to location (A) and solar zenith angle (B). (A) Effect of eclipse geometry on the altitudinal position of the umbra at three different locations at the same time. (B) Effect of eclipse geometry on the altitudinal extension of the umbra above a certain location at three different solar zenith angles $\left(60^{\circ}, 30^{\circ}\right.$ and $\left.10^{\circ}\right)$. Note that we refer to the zenith angle as measured at (the centre of) the intersection and not at ground level.

they can be observed as TIDs at ionospheric heights. Thus, AGW/TID effects can be detected in the electron distribution derived from the Digisonde soundings. Such detection is usually done by inspecting the so-called "true-height contours", i.e. the curves that are produced when plotting, over a certain time period, the reflection heights of ionosonde signals of different fixed frequencies. This reflection occurs at the altitude where the electron density is such that the plasma frequency is equal to the transmitted frequency. Therefore, a wave in plasma density will manifest itself in a plot of the true-height contours as a collective movement of multiple frequency curves (Gershman \& Grigorev 1968).

Presented here (Figs. 14C and 14D) are the true-height contours for several frequencies, ranging from $2.8 \mathrm{MHz}$ to $5.0 \mathrm{MHz}$, during the eclipse (Fig. 14C) and reference (Fig. 14D) days. The red arrows point at possible TID effects during the eclipse. Such peaks indicate the presence of TIDs only if there is a concurrent movement of all contours, and if the time scale of the variations is sufficiently longer than the repetition rate of the ionosonde soundings. The typical time scale for a TID is in the order of a few tens of minutes. Ionospheric soundings were obtained at 30-s intervals during the period of the eclipse, and at 5-min intervals during the rest of the day. Thus, abundant data were available to identify the indicated variations as TIDs. The TIDs seen during the eclipse were all "negative" density waves, which show up in the contour plots as upward peaks. The observed periods were between 10 and $15 \mathrm{~min}$.

From the true-height contours displayed here, the period and amplitude of a TID have been obtained but not the travelling direction and speed. Direction and speed can also be extracted but by using other capabilities of the Digisonde$4 \mathrm{D}$, the identification of the angle of arrival and the Doppler shift of each echo in addition to its range. Details of such measurements, carried out during the March 2015 eclipse, and their analysis can be found in the work by Verhulst et al. (2016).

\section{Eclipse geometry effects on the ionospheric response}

Here we will examine some effects that the eclipse geometry has on the ionosphere-plasmasphere system response to the eclipse. The standard, most popular way to look at the eclipse geometry effects is to consult the map of the eclipse on the Earth's surface (cf. Fig. 1) where the path of totality is given together with isolines of the gradually decreasing eclipse magnitude farther away from this path. Surface maps can be used to explain why during the eclipse the TEC drops much more (with reference to the same-day trend) near the totality path (Faroe Islands) than at faraway locations (e.g. Belgrade) as clearly seen in Figures $12 \mathrm{~A}, 12 \mathrm{D}$ and $12 \mathrm{G}$ at times close to the maximum eclipse, cf. the $t_{\mathrm{m}}$ marker. This is possible because the TEC is an integral characteristic (by definition, the integral of the electron density between two points along a slant/vertical path) of the total ionisation and as such does not "keep track" of how the ionisation is distributed spatially. However, it is not possible to explain from only the surface eclipse map, the observation that the relative TEC decrease (cf. Figs. 12C, $12 \mathrm{~F}$ and 12I), and the eclipse effects on the ionosphere in general, last differently at different locations and that the ionospheric recovery is (much) quicker near the totality path despite the larger obscuration of the Sun. To be able to explain these and the other observations presented in the previous sections, we need to also examine the positioning of the umbra (and penumbra) with reference to the different locations (Fig. 15).

It is clear from the figure that, at different locations, the umbra crosses the Earth's atmosphere at different altitudes (Fig. 15A). At location \#1, the umbra is entirely in the lowest part (i.e. in the troposphere/stratosphere), leaving the ionosphere only partially under the penumbra while the plasmasphere is sunlit and not directly affected by the eclipse. At location \#2, the umbra and the penumbra envelope the entire ionosphere with the umbra completely covering the altitude region of the peak plasma density (around $h_{\mathrm{m}} \mathrm{F}_{2}$ ). At location \#3, the umbra is entirely in the plasmasphere, i.e. above the upper ion transition level (UTL). Only the topside ionosphere is partially covered by the penumbra, while the region around $h_{\mathrm{m}} \mathrm{F}_{2}$ and the lower ionosphere are entirely exposed to the sunlight. Although the schematic is not to scale, the situation is similar to the one at the locations of the Faroes (\#1), Brussels (\#2) and Belgrade (\#3). Now, after taking into account the fact that most of the electron content comes from the region around $h_{\mathrm{m}} \mathrm{F}_{2}$, it becomes clear why the TEC relative deviation above Brussels (Fig. 12F) is largest and the relative deviation above Belgrade (Fig. 12I) is smallest. 
What is also evident from the figure is that, with the progress of the eclipse, the umbra crosses the Earth's atmosphere (at a given location) at different zenith angles (cf. Figs. 1C and 15B). Since the zenith angle varies with altitude, in the analysis here we refer only to the zenith angle at (the centre of) the intersection. For this eclipse, the zenith angles are larger above the Faroe Islands and smaller above Belgrade. The size of the intersection, denoted by the vertical dashed line, increases at smaller zenith angles (Fig. 15B). Considering the importance of the intersection's position in vertical direction, the size of this intersection will have a significant impact on TEC only at altitudes where it matters, i.e. in the ionosphere, at $h_{\mathrm{m}} \mathrm{F}_{2}$ in particular.

The eclipse geometry can also help in explaining (at least partially) why the TEC above the Faroes started decreasing just before the start of the eclipse (at ground level) and recovered much faster than at locations farther away from the totality path (Figs. 10-12). Along the totality path, the solar zenith angle was too large (i.e. small size of the intersection) and the altitude of the intersection was too low to have a substantial impact on TEC until the immediate arrival of the total eclipse. The recovery started shortly after the time of the maximum eclipse, and is much faster, again because of the larger zenith angle. Due to the smaller zenith angle above Belgrade, the size of the intersection was larger and contained the area around $h_{\mathrm{m}} \mathrm{F}_{2}$ for a longer period. Another reason for the slower recovery above Belgrade is that for this particular eclipse the southern stations experienced a much larger TEC decrease (in absolute terms, up to 14-15 TECU) than the northern stations. Recovering from such strong depletion of the ionosphere (usually) takes more time, the situation resembling the recovery after stronger geomagnetic storms.

It is very important to understand that the eclipse geometry changes with altitude. To demonstrate this, we have calculated three key eclipse characteristics - the zenith angle at the maximum eclipse, the obscuration level at the maximum eclipse and the time of the maximum eclipse - at the mean ionospheric height of $h_{\mathrm{m}}=300 \mathrm{~km}$. The results are presented in Figure 16 where the values at the $h_{\mathrm{m}}$ height (denoted with solid curves) are compared with the values at the sea level (denoted with dashed curves). The observed changes in geometry, going from sea level to ionospheric height, are highlighted by using (red) arrows. The first impression is that, at $h_{\mathrm{m}}$, all three maps appear to have diminished in sunward direction - most notably in the West (up to $10^{\circ}$ in longitude) and in the North (up to $5^{\circ}$ in latitude). As a result all three parameters (zenith angle, obscuration and time) appear differently on the map. The isolines are at new places and their shapes look different, particularly for the zenith angle and the time.

With regard to the zenith angle (Fig. 16A), the effect of changing altitude appears to be stronger in the middle of the isolines (i.e. along the SE-NW diagonal) and to gradually increase in NW direction. While the change in the South-East is negligible, the change in the North-West (e.g. the Faroes) is almost $5^{\circ}$ - the angle decreases from $70^{\circ}$ (at the time of the eclipse maximum) at sea level to about $65^{\circ}$ (at the time of the eclipse maximum) at ionospheric altitudes. As explained above, the smaller zenith angle of the umbra causes larger depletion in TEC, which combined with the stronger effect along the SE-NW diagonal, contributes to the formation and shape of the maximum $\mathrm{TEC}_{\text {rel }}$ depression area (Fig. 11).

It is obvious that the obscuration (Fig. 16B), at ionospheric altitudes, is increased in comparison of the obscuration at sea
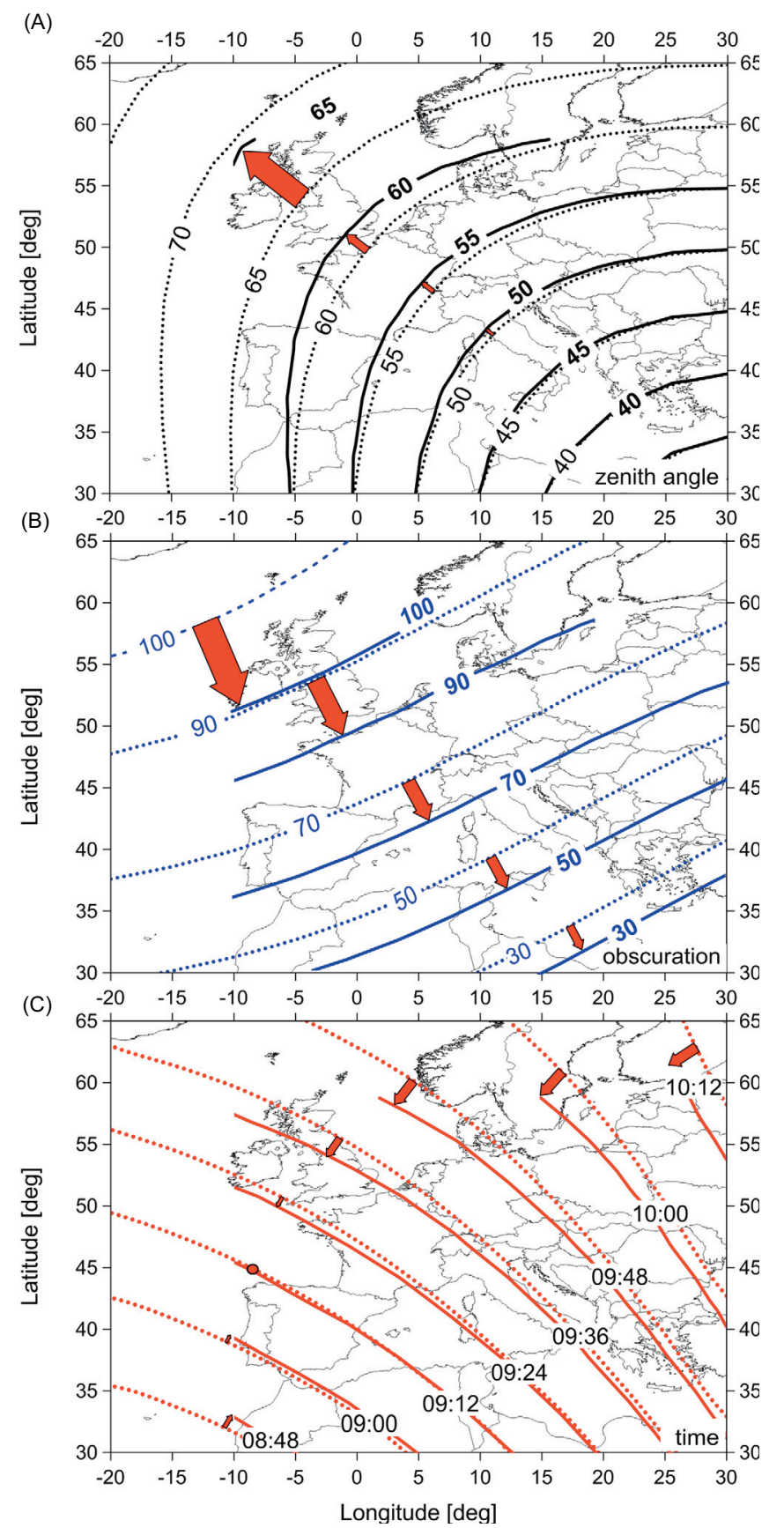

Fig. 16. Differences in eclipse geometry (Earth's curvature ignored) at ground level (dashed curves) and ionospheric height of $300 \mathrm{~km}$ (solid curves): zenith angle (A), obscuration (B), and time (C) at the eclipse maximum. The changes in geometry, going from sea level to ionospheric height, are highlighted by using (red) arrows.

level. The change is more or less uniform along the isolines. However, the effect is more pronounced in the North-West and less so in the South-East. Thus, in the ionosphere, the total obscuration has significantly shifted to cover also Ireland, Scotland, Norway, and parts of Sweden and Finland. Higher obscuration levels in the ionosphere should lead to larger depletions in the ionisation levels and the effect should increase in NW direction.

The time of the maximum eclipse (Fig. 16C) also changes with altitude and in a more complicated fashion. As with the zenith angle and the obscuration, the differences in the times 
(A)

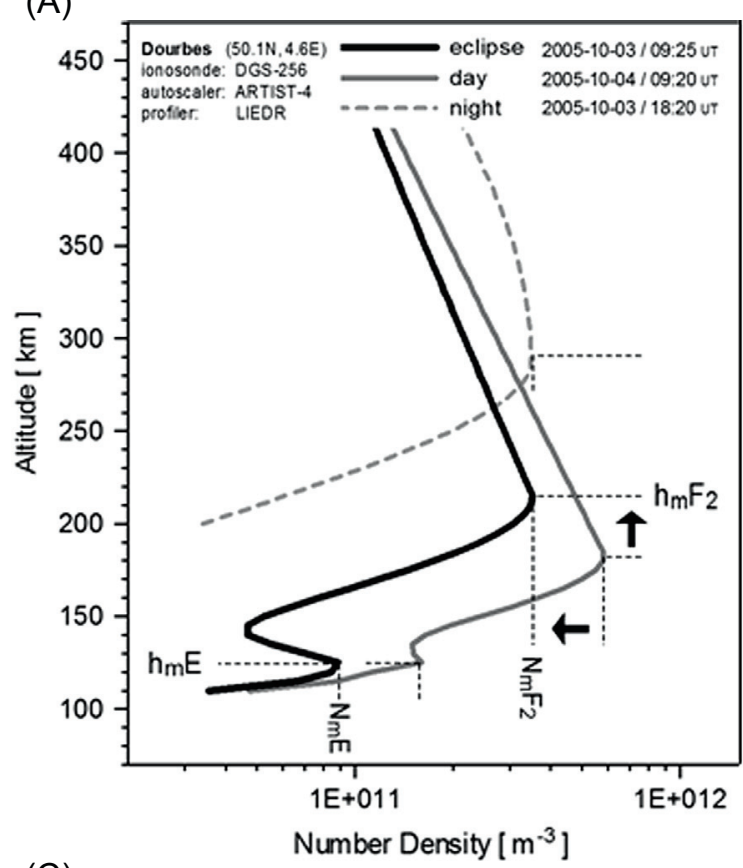

(C)

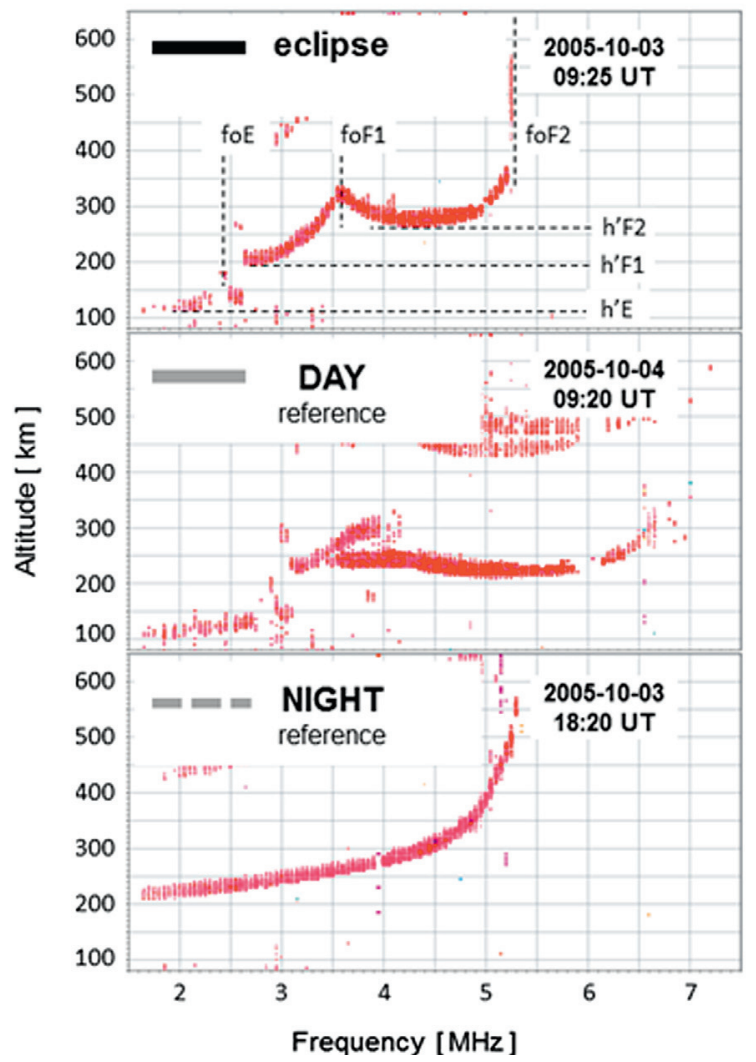

(B)

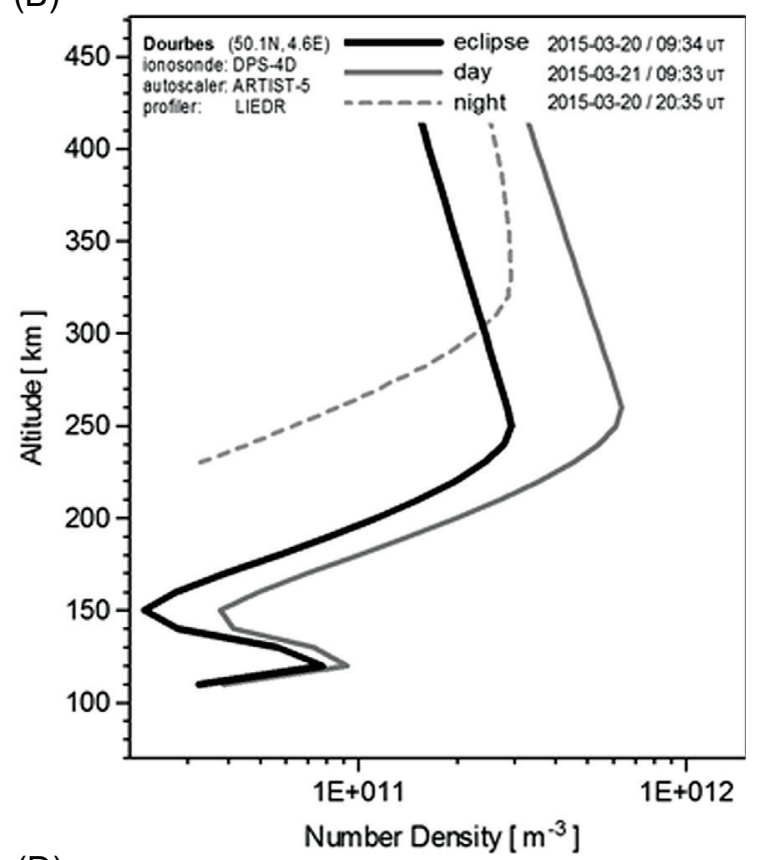

(D)

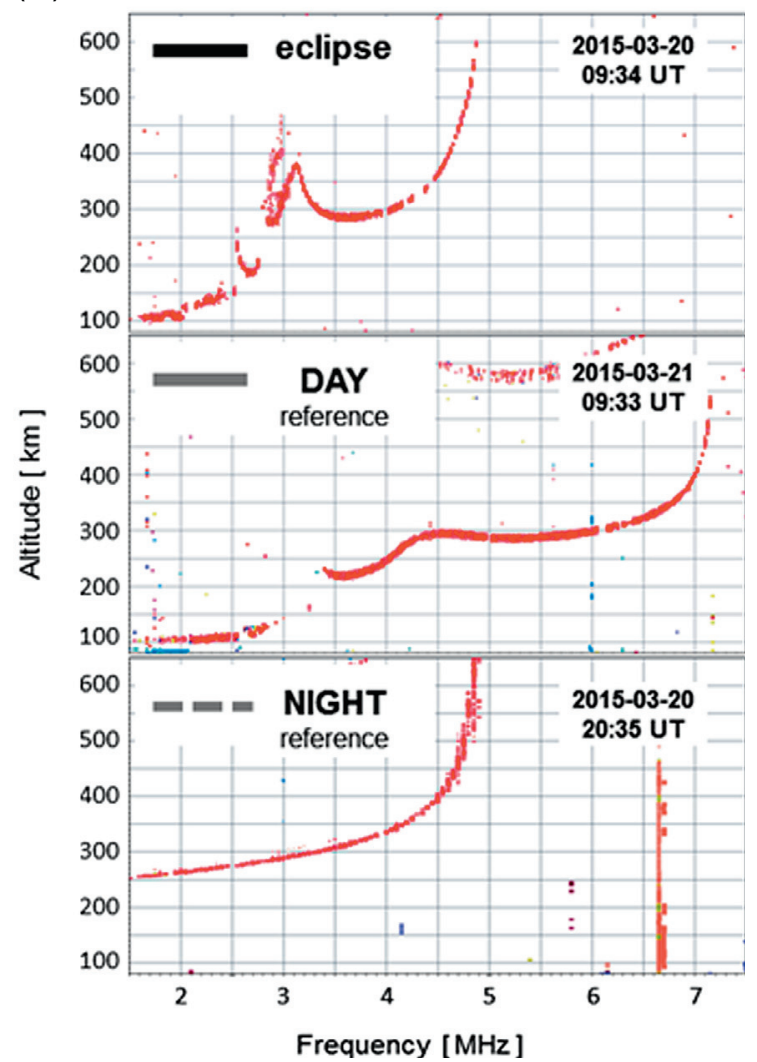

Fig. 17. Eclipse effects on the vertical distribution of the ionospheric electron density over Dourbes during the eclipses on 3 October 2005 (A) and 20 March 2015 (B). The electron density profile during the eclipse (black solid curve) is compared with a profile at the same time on a normal day (grey solid curve) and with a typical night-time profile (grey dashed curve). The ionograms used for the (LIEDR) reconstruction of the electron density profiles are displayed in (C) and (D).

at sea level and at higher altitudes increase in NW direction. In the initial phase of the eclipse, due to the Sun's elevation and azimuth, the maximum eclipse in the South-West appears on the ground before the maximum in the ionosphere (see the 08:48 UT and 09:00 UT isolines). At around 09:12 UT, the maximum eclipse occurs at the same time at different altitudes along the corresponding isoline. Afterwards, the maximum eclipse in the ionosphere lags the maximum on the ground, and this lag is increasing progressively in the later phases of the eclipse. This means that, around the time of the greatest eclipse at 09:45 UT, the shadow "travels" a much shorter distance for the same time period in the ionosphere than on the ground. In other words, the eclipse is "slower" in the ionosphere, which means that the ionosphere is exposed to the 

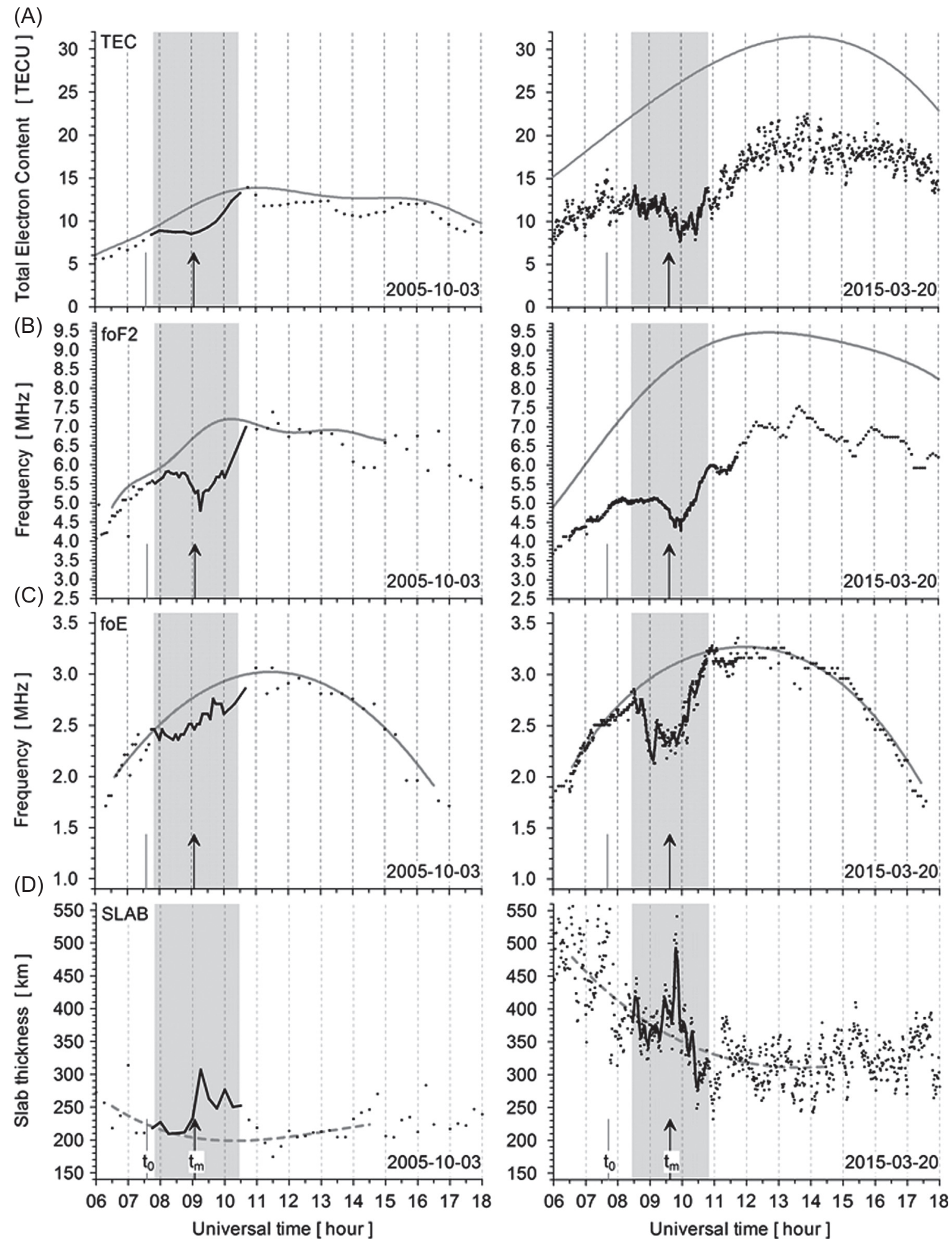

Fig. 18. (A-D) Comparison of the TEC, $f_{o} \mathrm{~F}_{2}, f_{o} \mathrm{E}$ and slab thickness behaviour during the eclipses of 3 October 2005 (left side) and 20 March 2015 (right side). The instantaneous measurements are denoted with black dots, the (approximated) daytime trend is plotted with a dashed grey curve and the (approximated) median behaviour with a solid grey curve. The shading covers the time period of the eclipse over Brussels and the measurements during the eclipse are highlighted with a solid black curve.

eclipse for a longer period than the ground. This, in combination with the changes in the zenith angle and obscuration levels at higher altitudes, can explain, e.g. the shape and location of the area of maximal TEC depletion observed in the TEC difference maps (Fig. 10C) and the maximum TEC difference map (Fig. 11A).
There are, of course, other mechanisms that can contribute to the different ionospheric responses at different locations such as, for example, the ionospheric plasma drifts (Verhulst et al. 2016). Unfortunately, there were no ionosonde drift measurements available from the Faroe Islands and Belgrade to be able to properly analyse the response across a wider area. 

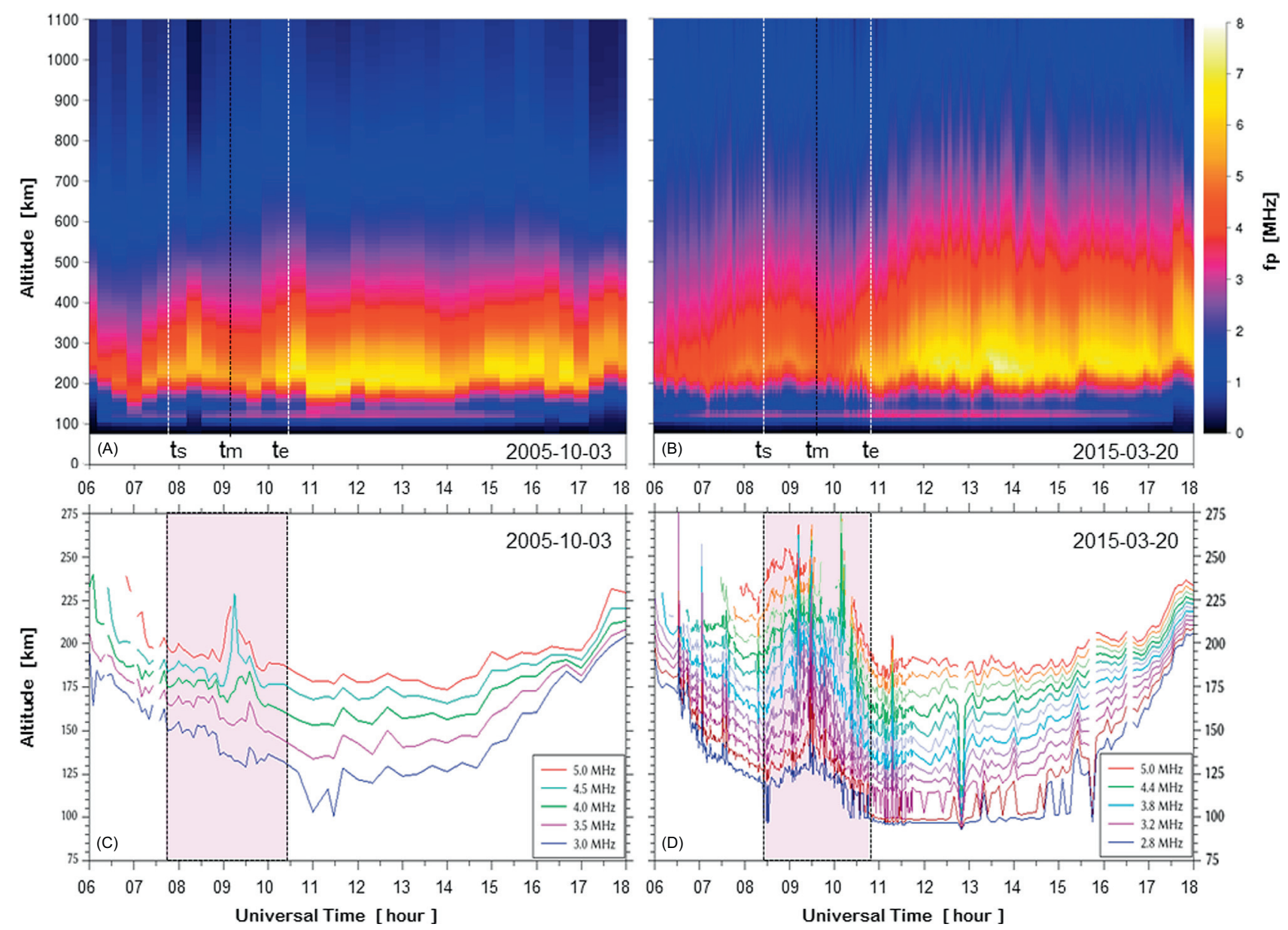

Fig. 19. Vertical plasma density distribution (A, B) and true-height contours (C, D) over Dourbes during the eclipses of 3 October 2005 (A, C) and 20 March 2015 (B, D). The true-height contours, displayed for frequencies ranging from $2.8 \mathrm{MHz}$ (bottom curve) up to $5.0 \mathrm{MHz}$ (top curve), are obtained from Digisonde measurements. The shadings indicate the eclipse periods. The time resolution is 15 min for the 2005 eclipse and $5 \mathrm{~min}$ for the 2015 eclipse, except between 06:30 UT and 11:30 UT on 20 March 2015 when the resolution is $30 \mathrm{~s}$.

\section{Comparison with ionospheric observations from the eclipse on 3 October 2005}

In this section, more details on the observations regarding the ionosphere during the March 2015 eclipse are provided and, whenever possible, a comparison is made with corresponding observations from the annular solar eclipse that occurred on 3 October 2005. That eclipse was first observed over the North Atlantic Ocean at 07:35 UT, the path crossed Portugal and Spain, the Mediterranean, Africa, and ended over the Indian Ocean at 13:28 UT. Over Brussels, the eclipse started at 07:51 UT, reached its maximum (magnitude about 68\%) at 09:04 UT and ended at 10:24 UT. Although the "geometry" of the two eclipses is different, there are similarities that justify a comparison of the observations. For example, both eclipses occurred at similar levels of solar activity, both occurred during equinox, at almost the same time in the morning, and the difference in magnitude (in Belgium) was only about $13 \%$.

First we will examine some ionograms, together with the reconstructed electron density profiles (Fig. 17) made at the eclipse maxima, and compare them with ionograms and profiles obtained during the reference days and nights. As demonstrated in the figure, there are obvious similarities between the eclipse and the night-time behaviour, e.g. the decrease of the peak electron densities and the increase of the peak height. However, although the decrease in the peak densities is similar in magnitude, the peak density height of the night-time F layer is way above the peak density height of the eclipsed ionosphere. Moreover, there is no E layer during night while it is still present during the eclipse.

Presented next is a more detailed comparison of (some of) the key ionospheric characteristics during both eclipses (Fig. 18). In Figures $18 \mathrm{~A}$ and $18 \mathrm{~B}$, right side, it can be seen that, during the 2015 eclipse, both TEC and $f_{o} \mathrm{~F}_{2}$ started to stagnate before the onset of the eclipse over the Dourbes observatory. This phenomenon was not seen during the 2005 eclipse. An explanation for these observations can be found in the difference in time between $t_{0}$ (the time of the first contact of the penumbra) and the start of the eclipse at the observation site $\left(t_{\mathrm{s}}\right)$. For the 2005 eclipse, the period between $t_{0}$ and the start of the eclipse over Belgium was only $16 \mathrm{~min}$, while for the 2015 eclipse this period was $46 \mathrm{~min}$. Therefore, during the 2015 eclipse, the ionosphere over the Atlantic Ocean was already in the shadow of the moon, well before the eclipse was observed locally in Dourbes. This induces gradients in the ionisation and the temperature, in turn resulting in bulk plasma movement towards the darkened region. During the 2005 eclipse, the time between $t_{0}$ and the local start of the eclipse was too short for this type of transport process to have a significant effect. Note that a similar precursory disturbance is not observed in the E-layer ionisation, represented here by $f_{o} \mathrm{E}$ (Fig. 18C), even for the 2015 case. This is understandable 
given the unimportance of transport processes in the E layer where the degree of ionisation is solely determined by the local solar irradiance and recombination.

Since the 2005 eclipse, significant improvements have been made in the observational instrumentation and the computer software used for the data analysis. Most importantly, the Digisonde-4D was installed in Dourbes in 2011, replacing the old sounder, Digisonde-256, which had been in continuous operation since 1984. The software used for the automatic interpretation (autoscaling) of ionograms was upgraded to the latest version, ARTIST-5. Also, a new NovAtel GPStation-6 GNSS receiver was installed in Dourbes, capable of providing real-time TEC at 60-s time resolution. This receiver is exclusively dedicated to providing measurements complementing the Digisonde measurements, whereas in 2005 only the data from the International GNSS Service (IGS) receiver was available, which had a time resolution of $15 \mathrm{~min}$ (Stankov et al. 2011). This combination of improvements in both the hardware and software units makes it now possible to produce reliable soundings at a much higher cadence, which in turn results in more accurate estimations of the changes in the ionosphere (Bamford 2001; Davis et al. 2001).

There are some important benefits from the increased sounding cadence. It can be seen from Figures 18A (TEC) and $18 \mathrm{~B}\left(f_{o} \mathrm{~F}_{2}\right)$ that the ionospheric disturbance due to the eclipse is a rather fast process, resulting in sharp peaks in the ionospheric characteristics. In 2005, when the observation rates were $15 \mathrm{~min}$ for the GNSS receiver and $5 \mathrm{~min}$ for the ionosonde, the disturbances could only be partially observed, which is obvious when looking at the smoother curves in the left side of Figures 18A-18D. In particular, the 15-min resolution of the TEC data is clearly inadequate for reliable observations of an eclipse. The higher time resolution also allows for the implementation of a simple data cleaning procedure replacing the outliers with interpolated values.

Higher-cadence observations also result in higher accuracy of the ionosphere monitoring systems and models based on these data. For example, in the slab thickness measurements (cf. Fig. 18D), the peak variability is much larger during the 2015 eclipse than during the 2005 eclipse. This can be (partially) explained by the difference in solar activity at the time of the respective eclipses which is certainly the reason for the different background trends. However, it is clear that the peak on the right side of Figure 18D is very sharp and could easily have been missed if using a coarser rate of observations. Similarly, the effects of the eclipse are less clear in the output of the LIEDR system for the 2005 eclipse (Fig. 19A) than for the 2015 eclipse (Fig. 19B).

Another result of the higher sounding rate is the possibility of observing travelling ionospheric disturbances (TIDs). TIDs typically have a period of 10-30 min, which means that they might be missed entirely when producing soundings at 15-min intervals. Even with a 5-min rate it can be difficult to separate the TID effects from the data noise. For the 2005 eclipse, when the soundings during the eclipse were done at 5-min intervals, it was not possible to unambiguously identify TID effects in the true-height contour plot (Fig. 19C). For the 2015 eclipse, a special campaign was run, producing ionograms at 30-s intervals (Verhulst et al. 2016). The TID effects, caused by the eclipse, are now clearly visible (Fig. 19D).

In addition to the traditional vertical ionospheric sounding producing ionograms, the Digisonde-4D also produces SkyMap observations. From these, the plasma drift and tilt of the ionosphere can be derived, providing a complementary method for the observation of wave phenomena (Verhulst et al. 2016).

\section{Summary}

A partial solar eclipse was observed over Belgium in the morning hours of 20 March 2015. The STCE observational campaign aimed at carrying out high-quality measurements that would help further our current understanding of the eclipse effects on the geospace environment, particularly on the local ionosphere. The results can be summarised as follows.

In order to better understand the physics behind a certain phenomenon like an eclipse, and the associated effects on the various environments such as the plasmasphere, ionosphere and atmosphere, multiple instruments of different types should be put in place to be able to perform a comprehensive analysis and to minimise the impact of instrument malfunction or unfavourable conditions. For this purpose, we have made use of several ground- and space-based instruments. Ground-based white-light coronal images during eclipses offer a way to observe the structure of the solar corona, allowing for mapping the density of free electrons. This in turn is a basic parameter for determining the coronal temperature and ion densities. In this particular instance, the eclipse was only partial at the ground-based STCE observatories, therefore no study on the white-light corona was possible. Nevertheless, space-based observations, carried out onboard the PROBA2 spacecraft, allowed picturing the structure of the corona in EUV. Images and flux measurements acquired during the campaign (even though the eclipse was partial) can now be used to validate models of solar irradiance in various wavelength ranges. They allow separating the respective contributions of the different solar structures from the general centre-to-limb variation. The effect of such structures was noticeable in the EUV and radio flux measurements during the eclipse.

The geomagnetic storm that started a few days before the eclipse had a substantial impact on the inner magnetosphere, the plasmasphere and the ionosphere. The ionosphere was not able to fully recover by the time of the eclipse, the total ionisation in particular. The lasting effects of the storm resulted in the behaviour of some ionospheric characteristics and variations to be dampened, perhaps causing the omission of some "low-profile" effects.

Apparently, the eclipse appeared to have little effect on the plasmasphere, neither on the density nor on the temperature, as deduced by the coupled ionosphere-plasmasphere modelling calculations. Even the day/night asymmetry was not particularly expressed in this region, in contrast to what happened at the lower, ionospheric altitudes.

Digital ionospheric soundings, carried out at a cadence of 30 s during the eclipse, allowed for the unambiguous identification of travelling ionospheric disturbances. The TIDs detected during this particular eclipse event appear to be electron density depletion waves with estimated periods of 10-15 min. From the true-height contours alone, the TID's period and amplitude could only be obtained; its direction and speed were determined in a parallel study utilising the ionosonde measurements of the angle of arrival and the Doppler shift of each sounding echo.

The high temporal and spatial resolution of the ionospheric observations is of great importance for carrying out a useful and 
reliable study of a quick-developing phenomenon like the eclipse. Over the last decade or two, the constant advancements in the observational instrumentation, combined with the progress made in computer hardware and software, now provide more possibilities for studying the eclipse effects. For example, thanks to the capabilities of the new digital ionosonde, we were able to execute an unprecedented high-resolution 30-s sounding campaign producing a good-quality autoscaled ionogram dataset. This proved very important for revealing some finer details in the reaction of the local ionosphere due to the eclipse. Another result made possible thanks to the higher sounding rate was the more reliable observation of wave phenomena and periodicities based on ionosonde measurements. Given their characteristic period of 10-30 minutes, TIDs might have been missed with soundings at 15 -min intervals, which was the norm until recently and would have been certainly missed out entirely with the 60-min norm not very long ago. High-resolution TEC measurements, available with a time resolution of $1 \mathrm{~min}$ for local data and $5 \mathrm{~min}$ for regional maps, were also important. Much more accurate estimations of the disturbances in the ionosphere, in the topside ionosphere in particular (e.g. the topside plasma redistribution processes), were made possible.

It is impossible to provide reliable interpretation of the eclipse effects on the ionosphere without carefully considering the eclipse geometry and its changes with time. Therefore, the geometry of this particular eclipse has been analysed and presented in great detail. The analysis covered not only the commonly referenced geographical position and extent of the totality path and the obscuration levels at the Earth's surface but also, and more importantly, the obscuration level, solar zenith angle and time of the maximum eclipse at ionospheric altitudes. Substantial and geographically irregular changes in the above-mentioned eclipse parameters have been revealed for different altitudes. This helped in explaining (at least partially) and estimating some of the eclipse effects (e.g. the delay in the plasma density decrease and recovery) and the different ionospheric reaction at different locations (e.g. the shape and location of the area of maximal electron content depletion).

Finally, some important conclusions can be drawn from this study with regard to the challenges and the interesting opportunities for future eclipse observations and research. Perhaps, the most important and challenging task is to ensure coordinated observations across several sites equipped with similar instruments; this is most relevant to observations of the ionosphere because there the effects of an eclipse are most dependent on the geographic location. Although it is possible to detect, for example, wave phenomena in the ionosphere at a single site, in order to track them and study their evolution with time, high-cadence observations at as many locations as possible along the wave paths are needed. Also, it has to be kept in mind that the eclipse geometry can be (very) different between eclipses, thus it is not always possible to determine whether an observed difference in the ionospheric reaction is due to varying eclipse onset time, obscuration level, geometry or background conditions. Therefore, for reliable analyses, building a comprehensive database of concurrent and diverse measurements is of utmost significance.

Acknowledgements. This study is funded by the Belgian SolarTerrestrial Centre of Excellence (STCE). V. Pierrard, D. Berghmans and E. D'Huys thank the Belgian Federal Scientific Policy Office (BELSPO) for the interuniversity project P7/08 CHARM. M. Dominique acknowledges the support from the Belgian Federal Science Policy Office (BELSPO) through the ESA-PRODEX programme. SWAP is an instrument developed by the Centre Spatial de Liège (CSL) and the Royal Observatory of Belgium (ROB), and funded by the Belgian Federal Science Policy Office (BELPSO). LYRA is a project of the Centre Spatial de Liège (CSL), the Physikalisch-Meteorologisches Observatorium Davos, and the Royal Observatory of Belgium (ROB), funded by the Belgian Federal Science Policy Office (BELPSO) and by the Swiss Bundesamt fur Bildung und Wissenschaft. S. Stankov, T. Verhulst and D. Sapundjiev acknowledge the (partial) funding by NATO via the Net-TIDE project (NATO-SPS-984894). Eclipse predictions were made by Fred Espenak, NASA's GSFC, and the US Naval Observatory (USNO) Solar Eclipse Computer. WIND and Advanced Composition Explorer (ACE) satellite measurements were provided by the US National Space Science Data Center (NSSDC). Solar radio flux index F10.7 was provided by the US National Oceanic and Atmospheric Administration (NOAA). Geomagnetic indices Dst and $K_{\mathrm{p}}$ were provided by the World Data Center for Geomagnetism, Kyoto. The editor thanks Petra Koucka Knizova, Bruno Zolesi and an anonymous referee for their assistance in evaluating this paper.

\section{References}

Altadill, D., J.G. Sole, and E.M. Apostolov. Vertical structure of a gravity wave like oscillation in the ionosphere generated by the solar eclipse of August 11, 1999. J. Geophys. Res., 106(A10), 21419-21428, 2001, DOI: 10.1029/2001JA900069.

Bamford, R.A. The effect of the 1999 total solar eclipse on the ionosphere. Phys. Chem. Earth Part C, 26(5), 373-377, 2001, DOI: 10.1016/S1464-1917(01)00016-2.

Bergeot, N., J.-M. Chevalier, C. Bruyninx, E. Pottiaux, W. Aerts, Q. Baire, J. Legrand, P. Defraigne, and W. Huang. Near real-time ionospheric monitoring over Europe at the Royal Observatory of Belgium using GNSS data. J. Space Weather Space Clim., 4, A31, 2014, DOI: $10.1051 / \mathrm{swsc} / 2014028$.

Berghmans, D., R.A.M. Van der Linden, P. Vanlommel, R. Warnant, A. Zhukov, et al. Solar activity - nowcasting and forecasting at the SIDC. Ann. Geophys., 23(9), 3115-3128, 2005, DOI: 10.5194/angeo-23-3115-2005.

Beynon, W.J.G., and G.M. Brown. Solar eclipses and the ionosphere, Pergamon Press, London, 1956.

Bruyninx, C., H. Habrich, W. Söhne, A. Kenyeres, G. Stangl, and C. Völksen. Enhancement of the EUREF permanent network services and products. In: S. Kenyon, M.C. Pacino, and U. Marti, Editors. Geodesy for Planet Earth, IAG Symposia Series, Springer-Verlag, Berlin, 136, 27-35, 2012,

DOI: $10.1007 / 978-3-642-20338-1 \_4$.

Chimonas, G., and C.O. Hines. Atmospheric gravity waves induced by a solar eclipse. J. Geophys. Res., 75(4), 857-875, 1970, DOI: $10.1029 / \mathrm{GM} 018 \mathrm{p} 0716$.

Clette, F., P. Cugnon, and A. Koeckelenbergh. Observations of the solar corona in polarized white light during the total solar eclipse of February, 16, 1980: Preliminary results. Sol. Phys., 98, 163-171, 1985, DOI: 10.1007/BF00177206.

Clette, F., P. Cugnon, and J.-R. Gabryl. Modelling the electron density distribution in the July 1991 solar corona. In: V. Rusin, P. Heinzel, and J.-C. Vial, Editors, Solar Coronal Structures. Proc. IAU Colloquium, Tatranska Lomnica, Slovakia, 14-20 September 1993, 535-539, 1994.

Davis, C.J., E.M. Clarke, R.A. Bamford, M. Lockwood, and S.A. Bell. Long term changes in EUV and X-ray emissions from the solar corona and chromosphere as measured by the response of the Earth's ionosphere during total solar eclipses from 1932 to 1999. Ann. Geophys., 19(3), 263-273, 2001,

DOI: 10.5194/angeo-19-263-2001.

Dominique, M., J.-F. Hochedez, W. Schmutz, I.E. Dammasch, A.I. Shapiro, M. Kretzschmar, A.N. Zhukov, D. Gillotay, Y. Stockman, and A. Ben Moussa. The LYRA instrument onboard PROBA2: description and in-flight performance. Sol. Phys., 286(1), 21-42, 2013, DOI: 10.1007/s11207-013-0252-5. 
Farges, T., J.C. Jodogne, R. Bamford, Y. Le Roux, F. Gauthier, P.M. Vila, D. Altadill, J.G. Sole, and G. Miro. Disturbances of the western European ionosphere during the total solar eclipse of 11 August 1999 measured by a wide ionosonde and radar network. J. Atmos. Sol. Terr. Phys., 63(9), 915-924, 2001, DOI: 10.1016/S1364-6826(00)00195-4.

Gabryl, J.-R., P. Cugnon, and F. Clette. Comparing the largescale coronal electron density distribution of the 1991 and 1994 solar eclipses. In: Proc. NATO Advanced Research Workshop on Theoretical and Observational Problems Related to Solar Eclipses, Bucharest, Romania, 1-5 June 1996, 73-76, 1997.

Gabryl, J.-R., P. Cugnon, and F. Clette. Polarization observations and results of the 1998 February 26th solar corona. Contr. Astron. Obs. Skalnate Pleso, 28(3), 216-223, 1999.

Galkin, I.A., G.M. Khmyrov, A.V. Kozlov, B.W. Reinisch, X. Huang, V.V. Paznukhov. The ARTIST 5. In: P. Song, J.C. Foster, M. Mendillo, and D. Bilitza, Editors. Radio Sounding and Plasma Physics, AIP Conf. Proc., 974, 150-159, 2008, DOI: $10.1063 / 1.2885024$.

Gershman, B.N., and G.I. Grigorev. Traveling ionospheric disturbances - a review. Radiophys. Quantum Electron., 11(1), 1-13, 1968, DOI: 10.1007/BF01033534.

Halain, J.P., D. Berghmans, D.B. Seaton, B. Nicula, A. De Groof, M. Mierla, A. Mazzoli, J.M. Defise, and P. Rochus. The SWAP EUV imaging telescope. Part II: in-flight performance and calibration. Sol. Phys., 286(1), 67-91, 2013,

DOI: $10.1007 / \mathrm{s} 11207-012-0183-6$.

Jakowski, N., S.M. Stankov, V. Wilken, C. Borries, D. Altadill, J. Chum, D. Buresova, P. Sauli, F. Hruska, and L.R. Cander. Ionospheric behavior over Europe during the solar eclipse of 3 October 2005. J. Atmos. Sol. Terr. Phys., 70(6), 836-853, 2008, DOI: $10.1016 /$ j.jastp.2007.02.016.

Jodogne, J.C., and S.M. Stankov. Ionosphere-plasmasphere response to geomagnetic storms studied with the RMI-Dourbes comprehensive database. Ann. Geophys., 45(5), 629-647, 2002, DOI: $10.4401 /$ ag-3529.

Klobuchar, J.A., and H.E. Whitney. Ionospheric electron content measurements during a solar eclipse. J. Geophys. Res., 70(5), 1254-1257, 1965, DOI: 10.1029/JZ070i005p01254.

Koeckelenbergh, A. 1973-1999 Historique des expéditions belges d'éclipses totales. Ciel et Terre, 115(2), 66-70, 1999.

Lemaire, J.F., and K. Stegen. Improved determination of the location of the temperature maximum in the corona. Sol. Phys., 291(12), 3659-3683, 2016 DOI: $10.1007 / \mathrm{s} 11207-016-1001-3$.

Müller-Wodarg, I.C.F., A.D. Aylward, and M. Lockwood. Effects of a mid-latitude solar eclipse on the thermosphere and ionosphere a modelling study. Geophys. Res. Lett., 25(20), 3787-3790, 1998, DOI: $10.1029 / 1998$ GL900045.
Neckel, H., and D. Labs. Solar Limb Darkening 1986-1990 ( $\lambda \lambda 303$ to $1099 \mathrm{~nm}$ ). Sol. Phys., 153(1-2), 91-114, 1994 ,

DOI: $10.1007 / \mathrm{BF} 00712494$

Pierrard, V., and K. Stegen. A three-dimensional dynamic kinetic model of the plasmasphere. J. Geophys. Res., 113(A10), A10209, 2008, DOI: 10.1029/2008JA013060.

Pierrard, V., and M. Voiculescu. The 3D model of the plasmasphere coupled to the ionosphere. Geophys. Res. Lett., 38(12), L12104, 2011, DOI: 10.1029/2011GL047767.

Reinisch, B.W., I.A. Galkin, G.M. Khmyrov, A.V. Kozlov, K. Bibl, et al. New Digisonde for research and monitoring applications. Radio Sci., 44(1), RS0A24, 2009, DOI: 10.1029/2008RS004115.

Rishbeth, H. Solar eclipses and ionospheric theory. Space Sci Rev., 8(4), 543-554, 1968, DOI: 10.1007/BF00175006.

Rishbeth, H. Eclipse effects in the ionosphere. Nature, 226, 1099-1100, 1970, DOI: 10.1038/2261099a0.

Sauli, P., P. Abry, J. Boska, and L. Duchayne. Wavelet characterisation of ionospheric acoustic and gravity waves occurring during the solar eclipse of August 11, 1999. J. Atmos. Sol. Terr. Phys., 68(3-5), 586-598, 2006, DOI: 10.1016/j.jastp.2005.03.024.

Sauli, P., S.G. Roux, P. Abry, and J. Boska. Acoustic-gravity waves during solar eclipses: detection and characterization using wavelet transforms. J. Atmos. Sol. Terr. Phys., 69(17-18), 2465-2484, 2007, DOI: 10.1016/j.jastp.2007.06.012.

Seaton, D.B., D. Berghmans, B. Nicula, J.P. Halain, A. De Groof, et al. The SWAP EUV imaging telescope. Part I: instrument overview and pre-flight testing. Sol. Phys., 286(1), 43-65, 2013, DOI: $10.1007 / \mathrm{s} 11207-012-0114-6$.

Shapiro, A.I., W. Schmutz, M. Dominique, and A.V. Shapiro. Eclipses Observed by Large Yield RAdiometer (LYRA) - a sensitive tool to test models for the solar irradiance. Sol. Phys., 286(1), 271-287, 2013, DOI: 10.1007/s11207-012-0063-0.

Stankov, S.M., and R. Warnant. Ionospheric slab thickness analysis, modelling and monitoring. Adv. Space Res., 44(11), 1295-1303, 2009, DOI: 10.1016/j.asr.2009.07.010.

Stankov, S.M., K. Stegen, P. Muhtarov, and R. Warnant. Local ionospheric electron density profile reconstruction in real time from simultaneous ground-based GNSS and ionosonde measurements. Adv. Space Res., 47(7), 1172-1180, 2011, DOI: $10.1016 /$ j.asr.2010.11.039.

Thuillier, G., T. Foujols, D. Bolsée, D. Gillotay, M. Hersé, et al. SOLAR/SOLSPEC: scientific objectives, instrument performance and its absolute calibration using a blackbody as primary standard source. Sol. Phys., 257(1), 187-213, 2009, DOI: $10.1007 / \mathrm{s} 11207-009-9361-6$

Verhulst, T.G.W., D. Sapundjiev, and S.M. Stankov. High-resolution ionospheric observations and modeling over Belgium during the solar eclipse of 20 March 2015 including first results of ionospheric tilt and plasma drift measurements. Adv. Space Res., 57(11), 2407-2419, 2016, DOI: 10.1016/j.asr.2016.03.009.

Cite this article as: Stankov SM, Bergeot N, Berghmans D, Bolsée D, Bruyninx C, et al. Multi-instrument observations of the solar eclipse on 20 March 2015 and its effects on the ionosphere over Belgium and Europe. J. Space Weather Space Clim., 7, A19, 2017, DOI: 10.1051/swsc/ 2017017. 